\title{
Extension of the Matrix Bartlett's Formula to the Third and Fourth Order and to Noisy Linear Models With Application to Parameter Estimation
}

\author{
Jean-Pierre Delmas, Member, IEEE, and Yann Meurisse
}

\begin{abstract}
This paper focuses on the extension of the asymptotic covariance of the sample covariance (denoted Bartlett's formula) of linear processes to third- and fourth-order sample cumulant and to noisy linear processes. Closed-form expressions of the asymptotic covariance and cross-covariance of the sample second-, third-, and fourth-order cumulants are derived in a relatively straightforward manner, thanks to a matrix polyspectral representation and a symbolic calculus akin to a high-level language. As an application of these extended formulae, we underscore the sensitivity of the asymptotic performance of estimated ARMA parameters by an arbitrary third- or fourth-order-based algorithm with respect to the signal-to-noise ratio, the spectra of the linear process, and the colored additive noise.
\end{abstract}

Index Terms-Bartlett's formula, fourth-order cumulant, noisy linear process, statistical performance analysis, third-order cumulant.

\section{INTRODUCTION}

$\mathbf{T}$ HE problem of estimating the parameters of linear time-invariant nonminimum phase systems when only output data are available from higher order statistics has been intensively studied. The use of the cumulants in time-series analysis has a long back history, starting with the classical paper of Brillinger and Rosenblatt [1] (see also Brillinger's book [2]). Giannakis [3] was the first to show that the parameters of a $q$ th-order MA system can be calculated from only the system's output cumulant with his third- and fourth-order formulae. From this pioneering work, many contributions have dealt with higher order statistics-based algorithms to estimate the MA, AR, and ARMA parameters of linear systems driven by an independent and identically distributed non-Gaussian sequence corrupted (or not) by additive Gaussian noise that may be colored (see, e.g., [4]-[8] and the reference therein).

The statistical performance of the proposed algorithms has been analyzed only by Monte Carlo simulations, except, to our knowledge, in the work by Porat and Friedlander [5] and by Dandawaté and Giannakis [9], [10]. This former work gives closed-form expressions for the asymptotic variances and covariances of the sample third-order moments of ARMA processes, thanks to a state-space representation focused on the

Manuscript received December 24, 2003; revised August 18, 2004. The associate editor coordinating the review of this manuscript and approving it for publication was Dr. Chong-Yung Chi.

The authors are with the GET/INT, Département CITI, UMR-CNRS 5157, Institut National des Télécommunications, 91011 Evry Cedex, France (e-mail: jean-pierre.delmas@int-evry.fr).

Digital Object Identifier 10.1109/TSP.2005.850362 noise-free case only. The latter work is dedicated to estimates of the asymptotic variances and covariances of sample $k$ th-order cumulants of arbitrary mixtures of deterministic, stationary, and nonstationary processes satisfying a mixing condition, based on smoothed cross periodograms.

The purpose of this paper is to give closed-form expressions of the asymptotic variances and covariances of the sample thirdand fourth-order cumulants of linear processes corrupted by an additive white or colored Gaussian or non-Gaussian noise. In addition, naturally, this paper provides tools for performance evaluation and comparison of identification algorithms based on sample third- or fourth-order cumulants in these conditions. The computation of each asymptotic variance/covariance in the noisy case turns out to be a very tedious task. For example, for zero-mean real-valued processes, the number of terms is 222 [resp. 6022] to express variance/covariance of the sample third-order [resp. fourth-order] moments in the noisy case to 41 [resp. 715] terms in the noise-free case. To overcome this computational difficulty, we propose in this paper to start from another point of view and to derive these variance/covariance via a matrix polyspectral approach. As a result, the complexity of the derivation of these different terms will not increase from the noise free to the noisy case. Furthermore, to avoid overly laborious calculations, we use a symbolic calculus akin to a high-level language.

This paper is organized as follows. After the data model and some notations are given in Section II, the second-order Bartlett's formula is recalled in Section III and expressed in a matrix polyspectral closed form in the noisy case for real-valued processes. This approach is extended in Sections IV and V to the third and fourth order, respectively. Because the derivation developed for the second order would be very tedious, a symbolic algorithm based on a few well-defined rules is used. Matrix closed-form expressions of the asymptotic covariance of the third-order sample moment and the asymptotic cross-covariance between the second- and third-order sample moments are given in the noisy case for zero-mean real-valued processes in Section IV. For the fourth order, we focus on zero-mean complex processes circular up to the fourth order as examples in Section $\mathrm{V}$, where we get closed-form expressions of the asymptotic covariance of the second- and fourth-order sample cumulants and the asymptotic cross-covariances between the second- and fourth-order sample cumulants in the noisy case. Finally, the sensitivity of the asymptotic performance of the estimated ARMA parameters by an arbitrary third- or fourth-order-based algorithm to the signal-to-noise ratio (SNR), 
the spectra of the linear process, and the colored additive noise is addressed in Section VI. As an example, the asymptotic lower bound for the variances of third- or fourth-order algorithms are compared to the asymptotic variances given by the so-called $C(k, q)$ algorithms for non-Gaussian first- or second-order moving average processes [abbreviated as $\mathrm{MA}(1)$ and $\mathrm{MA}(2)$ in the sequel] corrupted by a Gaussian first-order autoregressive process [abbreviated as AR(1) in the sequel].

The following notations are used throughout the paper. The range of all summations is understood to be from $-\infty$ to $\infty$, except the specified summations and the range of all integrations is $\triangle=[-1 / 2,1 / 2]$. $\operatorname{Cov}(\mathbf{x}, \mathbf{y})$ is used for real and complexvalued random vectors and means $\mathrm{E}\left(\mathrm{xy}^{T}\right)-\mathrm{E}(\mathbf{x}) \mathrm{E}\left(\mathbf{y}^{T}\right)$.

\section{DATA MODEL}

Consider the following linear process:

$$
x_{t}=\sum_{n} h_{n} u_{t-n}
$$

where $h_{n}$ is real valued in Sections III and IV [resp. complex valued in Section V] with $\sum_{n}\left|h_{n}\right|<\infty$, and the observation of $x_{t}$ is noisy

$$
y_{t}=x_{t}+\epsilon_{t}
$$

The input sequence $u_{t}$ is zero-mean, independent and identically distributed, and non-Gaussian, real-valued with $\kappa_{3_{u}} \stackrel{\text { def }}{=} \mathrm{E}\left(u_{t}^{3}\right) \neq 0$ and $\mathrm{E}\left(u_{t}^{6}\right)<\infty$ in Sections III and IV [resp. complex valued, circular up to the fourth order with $\kappa_{4_{u}} \stackrel{\text { def }}{=} \mathrm{E}\left|u_{t}^{4}\right|-2\left(\mathrm{E}\left|u_{t}^{2}\right|\right)^{2} \neq 0$ and $\mathrm{E}\left|u_{t}\right|^{8}<\infty$ in Section V]. The measurement noise sequence $\epsilon_{t}$ is assumed to be zero-mean, colored stationary with unknown power spectrum and is independent of $u_{t}$. In Sections III and IV, $\epsilon_{t}$ is real-valued Gaussian or non-Gaussian, with $\mathrm{E}\left(\epsilon_{t}^{2}\right) \stackrel{\text { def }}{=} \sigma_{\epsilon}^{2}$ and with sixth-order cumulants $c_{t_{2}-t_{1}, \ldots, t_{6}-t_{1}}^{\epsilon} \stackrel{\text { def }}{=} \operatorname{Cum}\left(\epsilon_{t_{1}}, \ldots, \epsilon_{t_{6}}\right)$ satisfying $\sum_{t_{1}} \ldots \sum_{t_{5}}\left|c_{t_{1}, t_{2}, \ldots, t_{5}}^{\epsilon}\right|<\infty$. So, the polyspectra of $y_{t}, x_{t}$, and $\epsilon_{t}$ are defined up to the fifth order

$$
S_{\epsilon}\left(f_{1}, f_{2}, \ldots, f_{p}\right) \stackrel{\text { def }}{=} \sum_{t_{1}} \ldots \sum_{t_{p}} c_{t_{1}, \ldots, t_{p}}^{\epsilon} e^{-i 2 \pi\left(t_{1} f_{1}+\ldots+t_{p} f_{p}\right)} .
$$

In Sections $\mathrm{V}, \epsilon_{t}$ is circular complex valued with $\mathrm{E}\left|\epsilon_{t}^{2}\right| \stackrel{\text { def }}{=}$ $\sigma_{\epsilon}^{2}$ and with eighth-order cumulants $c_{t_{2}-t_{1}, \ldots, t_{8}-t_{1}} \stackrel{\text { def }}{=}$ $\operatorname{Cum}\left(\epsilon_{t_{1}}, \epsilon_{t_{2}}^{*}, \ldots, \epsilon_{t_{2 k-1}}, \epsilon_{t_{2 k}}^{*} \ldots, \epsilon_{t_{8}}^{*}\right)$ satisfying $\sum_{t_{1}} \ldots \sum_{t_{7}}$ $\left|c_{t_{1}, t_{2}, \ldots, t_{7}}^{\epsilon}\right|<\infty$. So, the polyspectra of $y_{t}, x_{t}$ and $\epsilon_{t}$ are defined up to the seventh order

$$
\begin{aligned}
S_{\epsilon}\left(f_{1}, f_{2}, \ldots, f_{2 p-1}\right) \stackrel{\text { def }}{=} \sum_{t_{1}} \ldots & \sum_{t_{2 p-1}} c_{t_{1}, \ldots, t_{2 p-1}}^{\epsilon} \\
& \times e^{-i 2 \pi\left(t_{1} f_{1}+\ldots+t_{2 p-1} f_{2 p-1}\right)} .
\end{aligned}
$$

In Sections III and IV, the second-order moment $c_{k}^{x} \stackrel{\text { def }}{=}$ $\mathrm{E}\left(x_{t} x_{t+k}\right)$ and third-order moment $c_{k, l}^{x} \stackrel{\text { def }}{=} \mathrm{E}\left(x_{t} x_{t+k} x_{t+l}\right)$ are estimated from $T$ consecutive measurements by the associated sample moments: $c_{k}^{x}(T) \stackrel{\text { def }}{=}(1 / T) \sum_{t=1}^{T} x_{t} x_{t+k}$ and $c_{k, l}^{x}(T) \stackrel{\text { def }}{=}(1 / T) \sum_{t=1}^{T} x_{t} x_{t+k} x_{t+l} \cdot{ }^{1}$ These moments are stacked in the vectors $\mathbf{c}_{2}^{x} \stackrel{\text { def }}{=}\left(c_{0}^{x}, \ldots, c_{L_{2}-1}^{x}\right)^{T}$, $\mathbf{c}_{3}^{x} \stackrel{\text { def }}{=} \quad\left(c_{0,0}^{x}, c_{0,1}^{x}, \ldots, c_{0, L_{3}-1}^{x}, c_{1,0}^{x}, \ldots, c_{L_{3}-1, L_{3}-1}^{x}\right)^{T}$, $\mathbf{c}_{2}^{x}(T)$, and $\mathbf{c}_{3}^{x}(T)$ are defined in the same way. In Section $\mathrm{V}$, the second-order moment $c_{k}^{x} \stackrel{\text { def }}{=} \mathrm{E}\left(x_{t} x_{t+k}^{*}\right)$ and fourth-order cumulant $c_{k, l, m}^{x} \stackrel{\text { def }}{=} \mathrm{E}\left(x_{t} x_{t+k}^{*} x_{t+l} x_{t+m}^{*}\right)-$ $\mathrm{E}\left(x_{t} x_{t+k}^{*}\right) \mathrm{E}\left(x_{t+l} x_{t+m}^{*}\right)-\mathrm{E}\left(x_{t} x_{t+m}^{*}\right) \mathrm{E}\left(x_{t+l} x_{t+k}^{*}\right)$ are estimated from $T$ consecutive measurements by the associated sample cumulants: $c_{k}^{x}(T)$ and $c_{k, l, m}^{x}(T)$. These cumulants are stacked in increasing order in the vectors $\mathbf{c}_{4}^{x} \stackrel{\text { def }}{=}\left(c_{0,0,0}^{x}, c_{0,0,1}^{x}, \ldots, c_{L_{4}-1, L_{4}-1, L_{4}-1}^{x}\right)^{T}$, and $\mathbf{c}_{4}^{x}(T)$ is defined in the same way.

\section{SECOND-ORDER BARTLETT's Formula}

\section{A. Noise-Free Case}

Under the above assumptions, $\mathbf{c}_{2}^{x}(T)$ is asymptotically Gaussian (see, e.g., [11, Th. 3.3])

$$
\sqrt{T}\left(\mathbf{c}_{2}^{x}(T)-\mathbf{c}_{2}^{x}\right) \stackrel{\mathcal{L}}{\rightarrow} \mathcal{N}\left(\mathbf{0} ; \mathbf{C}_{2,2}^{x}\right)
$$

where $\stackrel{\mathcal{L}}{\rightarrow}$ stands for convergence in distribution, and $\mathbf{C}_{2,2}^{x}=$ $\lim _{T \rightarrow \infty} T \operatorname{Cov}\left(\mathbf{c}_{2}^{x}(T), \mathbf{c}_{2}^{x}(T)\right)$ is given by Bartlett's formula (see, e.g., [12, rel. 6, p. 255])

$$
\begin{aligned}
\lim _{T \rightarrow \infty} T \operatorname{Cov}\left(c_{k}^{x}(T), c_{l}^{x}(T)\right) \\
=\sum_{t}\left(c_{t}^{x} c_{t+k-l}^{x}+c_{t+k}^{x} c_{t-l}^{x}+c_{t+k, t, l}^{x}\right) .
\end{aligned}
$$

Using Parseval's theorem and the Fourier relationship between the covariance and the spectral density $S_{x}(f)$ of $x_{t}$ and between the fourth-order cumulant and the trispectrum $S_{x}\left(f_{1}, f_{2}, f_{3}\right)$ of $x_{t}$, we get the following alternative matrix polyspectral Bartlett's formula:

$$
\begin{aligned}
\mathbf{C}_{2,2}^{x}=\int_{\triangle} S_{x}^{2}(f) & {\left[\mathbf{e}(f) \mathbf{e}^{H}(f)+\mathbf{e}(f) \mathbf{e}^{T}(f)\right] d f } \\
& +\int_{\triangle^{2}} S_{x}\left(f_{1},-f_{1}, f_{2}\right) \mathbf{e}\left(f_{1}\right) \mathbf{e}^{T}\left(f_{2}\right) d f_{1} d f_{2}
\end{aligned}
$$

with $\mathbf{e}(f) \stackrel{\text { def }}{=}\left(1, e^{i 2 \pi f}, \ldots, e^{i 2 \pi\left(L_{2}-1\right) f}\right)^{T}$, where $T$ and $H$ stand for transpose and conjugate transpose, respectively. We note that an element-wise counterpart of this relation was derived in [2, rel. 5.10.15] by another approach.

\section{B. Noisy Case}

Under the assumptions of Section II, the asymptotic normality and (3.1) apply in the noisy case, by replacing $x_{t}$ by $y_{t}=x_{t}+\epsilon_{t}$. Furthermore, from the independence assumption, $S_{y}(f)=S_{x}(f)+S_{\epsilon}(f)$ and

${ }^{1}$ We note that both $c_{k}^{x}(T)$ and $c_{k, l}^{x}(T)$ can be defined in several other ways, differing in the manner in which the end data are treated. However, all of these definitions are asymptotically equivalent. 
$S_{y}\left(f_{1}, f_{2}, f_{3}\right)=S_{x}\left(f_{1}, f_{2}, f_{3}\right)+S_{\epsilon}\left(f_{1}, f_{2}, f_{3}\right)$, and thus, there holds

$$
\begin{aligned}
\mathbf{C}_{2,2}^{y}= & \mathbf{C}_{2,2}^{x}+2 \int_{\triangle} S_{\epsilon}(f) S_{x}(f)\left[\mathbf{e}(f) \mathbf{e}^{H}(f)+\mathbf{e}(f) \mathbf{e}^{T}(f)\right] d f \\
& +\int_{\triangle} S_{\epsilon}^{2}(f)\left[\mathbf{e}(f) \mathbf{e}^{H}(f)+\mathbf{e}(f) \mathbf{e}^{T}(f)\right] d f \\
& +\int_{\triangle^{2}} S_{\epsilon}\left(f_{1},-f_{1}, f_{2}\right) \mathbf{e}\left(f_{1}\right) \mathbf{e}^{T}\left(f_{2}\right) d f_{1} d f_{2} .
\end{aligned}
$$

We note that the last term of (3.2) vanishes if the additive noise is Gaussian.

\section{THIRD-ORDER BARTLETT'S FORMULA}

\section{A. Noise-Free Case}

Under the assumptions of Section II, $\left[\mathbf{c}_{2}^{x}(T), \mathbf{c}_{3}^{x}(T)\right]$ is asymptotically Gaussian (see, e.g., [1])

$$
\sqrt{T}\left(\begin{array}{l}
\mathbf{c}_{2}^{x}(T)-\mathbf{c}_{2}^{x} \\
\mathbf{c}_{3}^{x}(T)-\mathbf{c}_{3}^{x}
\end{array}\right) \stackrel{\mathcal{L}}{\rightarrow} \mathcal{N}\left(\left(\begin{array}{l}
\mathbf{0} \\
\mathbf{0}
\end{array}\right) ;\left(\begin{array}{cc}
\mathbf{C}_{2,2}^{x} & \mathbf{C}_{2,3}^{x} \\
\mathbf{C}_{2,3}^{x} T & \mathbf{C}_{3,3}^{x}
\end{array}\right)\right)
$$

where $\mathbf{C}_{2,3}^{x}=\lim _{T \rightarrow \infty} T \operatorname{Cov}\left(\mathbf{c}_{2}^{x}(T), \mathbf{c}_{3}^{x}(T)\right)$ and $\mathbf{C}_{3,3}^{x}=$ $\lim _{T \rightarrow \infty} T \operatorname{Cov}\left(\mathbf{c}_{3}^{x}(T), \mathbf{c}_{3}^{x}(T)\right)$, and it is straightforward to get (see, e.g., [13, rel.10.5.2])

$$
\begin{aligned}
& \lim _{T \rightarrow \infty} T \operatorname{Cov}\left(c_{k}^{x}(T), c_{l, m}^{x}(T)\right) \\
& \quad=\sum_{t}\left[\mathrm{E}\left(x_{0} x_{k} x_{t} x_{t+l} x_{t+m}\right)-c_{k}^{x} c_{l, m}^{x}\right] \\
& \lim _{T \rightarrow \infty} T \operatorname{Cov}\left(c_{k, l}^{x}(T), c_{m, n}^{x}(T)\right) \\
& \quad=\sum_{t}\left[\mathrm{E}\left(x_{0} x_{k} x_{l} x_{t} x_{t+m} x_{t+n}\right)-c_{k, l}^{x} c_{m, n}^{x}\right] .
\end{aligned}
$$

To proceed, deducing relations similar to (3.1) along the same lines is possible in principle, but such a derivation would be extremely tedious. A much more interesting approach, which consists of devising a symbolic calculus akin to a high-level language, is used. Based on a few well-defined rules, this algorithm allows us to automatically perform the following steps.

- Generate all partitions given by the cumulants-to-moments formula (Leonov Shiryaev formula) [13, Th. 10.1] expressing the fifth- $\left(\mathrm{E}\left(x_{0} x_{k} x_{t} x_{t+l} x_{t+m}\right)\right.$ of (4.1)) and sixth-order moments $\left(\mathrm{E}\left(x_{0} x_{k} x_{l} x_{t} x_{t+m} x_{t+n}\right)\right.$ of (4.2)) as functions of sums of products of cumulants.

- Eliminate the zero terms using the zero-mean property.

- Construct sets of similar expressions w.r.t. the number of terms $x_{t+a}$ in each product of cumulants. For each such set of similar expressions [three sets for $\mathrm{E}\left(x_{0} x_{k} x_{t} x_{t+l} x_{t+m}\right)$ and six sets for $\left.\mathrm{E}\left(x_{0} x_{k} x_{l} x_{t} x_{t+m} x_{t+n}\right)\right]$, a representative term is chosen to be analytically expressed by a polyspectral formula, as proved in Appendix A.

Consequently, using the (.) notation introduced in [14] to avoid listing explicitly all the partitions, we obtain

$$
\begin{aligned}
\mathrm{E}\left(x_{0} x_{k} x_{t} x_{t+l} x_{t+m}\right) & \\
= & \operatorname{Cum}\left(x_{0}, x_{k}, x_{t}, x_{t+l}, x_{t+m}\right) \\
& +\operatorname{Cum}\left(x_{0}, x_{k}, x_{t}\right) \operatorname{Cum}\left(x_{t+l}, x_{t+m}\right)(10)
\end{aligned}
$$

$$
\begin{aligned}
\mathrm{E}\left(x_{0}\right. & \left.x_{k} x_{l} x_{t} x_{t+m} x_{t+n}\right) \\
= & \operatorname{Cum}\left(x_{0}, x_{k}, x_{l}, x_{t}, x_{t+m}, x_{t+n}\right) \\
& +\operatorname{Cum}\left(x_{0}, x_{k}, x_{l}, x_{t}\right) \operatorname{Cum}\left(x_{t+m}, x_{t+n}\right)(15) \\
& +\operatorname{Cum}\left(x_{0}, x_{k}, x_{l}\right) \operatorname{Cum}\left(x_{t}, x_{t+m}, x_{t+n}\right)(10) \\
& +\operatorname{Cum}\left(x_{0}, x_{k}\right) \operatorname{Cum}\left(x_{l}, x_{t}\right) \\
& \times \operatorname{Cum}\left(x_{t+m}, x_{t+n}\right)(15) .
\end{aligned}
$$

As an example, the expression $\operatorname{Cum}\left(x_{0}, x_{k}, x_{t}\right) \operatorname{Cum}\left(x_{t+l}\right.$, $\left.x_{t+m}\right)(10)$ can be broken down into three sets

$$
\begin{aligned}
\operatorname{Cum} & \left.x_{0}, x_{k}, x_{t}\right) \operatorname{Cum}\left(x_{t+l}, x_{t+m}\right)(10) \\
= & c_{t+m-k}^{x} c_{l,-t}^{x}+c_{t+l-k}^{x} c_{m,-t}^{x}+c_{t}^{x} c_{m-l,-t+k-l}^{x} \\
& +c_{t-k}^{x} c_{m-l,-t-l}^{x}+c_{t+l}^{x} c_{m,-t-k}^{x}+c_{t+m}^{x} c_{l,-t+k}^{x} \\
& +c_{m-l}^{x} c_{k, t}^{x}+c_{m}^{x} c_{k, t+l}^{x}+c_{l}^{x} c_{k, t+m}^{x}+c_{k}^{x} c_{l, m}^{x}
\end{aligned}
$$

and the last term cancels with $-c_{k}^{x} c_{l, m}^{x}$ in summation (4.1). So, this expression reduces to two sets of similar expressions. Using expressions (4.1), (4.3), and (4.4), the following matrix polyspectral extensions of Bartlett's formula is proved in Appendix A, where each polyspectral integral is associated with a set of the previous similar expressions

$$
\begin{aligned}
& \lim _{T \rightarrow \infty} T \operatorname{Cov}\left(\mathbf{c}_{2}^{x}(T), \mathbf{c}_{3}^{x}(T)\right) \\
& =\int_{\triangle^{3}} S_{x}\left(f_{1},-f_{1}, f_{2}, f_{3}\right) \mathbf{E}_{1,2,3} d f_{1} d f_{2} d f_{3} \\
& \quad+\int_{\triangle^{2}} S_{x}\left(f_{1}, f_{2}\right) S_{x}\left(f_{1}\right) \mathbf{E}_{1,2}^{(1)} d f_{1} d f_{2} \\
& \quad+\int_{\triangle^{2}} S_{x}\left(f_{1}, 0\right) S_{x}\left(f_{2}\right) \mathbf{E}_{1,2}^{(2)} d f_{1} d f_{2}
\end{aligned}
$$

where matrices $\mathbf{E}_{1,2,3}, \mathbf{E}_{1,2}^{(1)}$, and $\mathbf{E}_{1,2}^{(2)}$ are defined in Appendix B.

With the same procedure, the following matrix polyspectral formula is derived from (4.2)

$$
\begin{aligned}
\lim _{T \rightarrow \infty} T \operatorname{Cov}\left(\mathbf{c}_{3}^{x}(T), \mathbf{c}_{3}^{x}(T)\right) & =\int_{\triangle^{4}} S_{x}\left(f_{1}, f_{2}, f_{3}, f_{4},-f_{3}-f_{4}\right) \mathbf{E}_{1,2,3,4} d f_{1} d f_{2} d f_{3} d f_{4} \\
& +\int_{\triangle^{3}} S_{x}\left(f_{1}, f_{3}\right) S_{x}\left(f_{2}, f_{3}\right) \mathbf{E}_{1,2,3}^{(1)} d f_{1} d f_{2} d f_{3} \\
& +\int_{\triangle^{3}} S_{x}\left(f_{1}, f_{2}, 0\right) S_{x}\left(f_{3}\right) \mathbf{E}_{1,2,3}^{(2)} d f_{1} d f_{2} d f_{3} \\
& +\int_{\triangle^{3}} S_{x}\left(f_{1}, f_{2}, f_{3}\right) S_{x}\left(f_{2}+f_{3}\right) \mathbf{E}_{1,2,3}^{(3)} d f_{1} d f_{2} d f_{3} \\
& +\int_{\triangle^{2}} S_{x}(0) S_{x}\left(f_{1}\right) S_{x}\left(f_{2}\right) \mathbf{E}_{1,2}^{(3)} d f_{1} d f_{2} \\
& +\int_{\triangle^{2}} S_{x}\left(f_{1}\right) S_{x}\left(f_{2}\right) S_{x}\left(f_{1}+f_{2}\right) \mathbf{E}_{1,2}^{(4)} d f_{1} d f_{2}
\end{aligned}
$$

where the different matrices $\mathbf{E}_{j}^{(i)}$ are defined in Appendix B. 


\section{B. Noisy Case}

In the noisy case, the third-order relations are derived in the same way as for the second order, because the independence of $x_{t}$ and $\epsilon_{t}$ ensures the additivity of their polyspectra. Therefore, for example, for additive Gaussian noise, we get from (4.5) and (4.6)

$$
\begin{aligned}
\mathbf{C}_{2,3}^{y}= & \mathbf{C}_{2,3}^{x}+\int_{\triangle^{2}} S_{\epsilon}\left(f_{2}\right) S_{x}\left(f_{1}, 0\right) \mathbf{E}_{1,2}^{(1)} d f_{1} d f_{2} \\
& +\int_{\triangle^{2}} S_{\epsilon}\left(f_{1}\right) S_{x}\left(f_{1}, f_{2}\right) \mathbf{E}_{1,2}^{(2)} d f_{1} d f_{2}
\end{aligned}
$$

$$
\mathbf{C}_{3,3}^{y}=\mathbf{C}_{3,3}^{x}+\int_{\triangle^{3}} S_{\epsilon}\left(f_{2}+f_{3}\right) S_{x}\left(f_{1}, f_{2}, f_{3}\right) \mathbf{E}_{1,2,3}^{(3)} d f_{1} d f_{2} d f_{3}
$$$$
+\int_{\triangle^{3}} S_{\epsilon}\left(f_{3}\right) S_{x}\left(f_{1}, f_{2}, 0\right) \mathbf{E}_{1,2,3}^{(3)} d f_{1} d f_{2} d f_{3}
$$$$
+\int_{\triangle^{2}}\left[S_{\epsilon}\left(f_{2}\right) S_{x}(0) S_{x}\left(f_{1}\right)+S_{\epsilon}\left(f_{1}\right) S_{x}(0) S_{x}\left(f_{2}\right)\right.
$$$$
\begin{gathered}
\left.+S_{\epsilon}(0) S_{x}\left(f_{1}\right) S_{x}\left(f_{2}\right)\right] \mathbf{E}_{1,2}^{(3)} d f_{1} d f_{2} \\
+\int_{\triangle^{2}}\left[S_{\epsilon}\left(f_{1}\right) S_{\epsilon}\left(f_{2}\right) S_{x}(0)+S_{\epsilon}(0) S_{\epsilon}\left(f_{2}\right) S_{x}\left(f_{1}\right)\right.
\end{gathered}
$$$$
\begin{gathered}
\left.+S_{\epsilon}(0) S_{\epsilon}\left(f_{1}\right) S_{x}\left(f_{2}\right)\right] \mathbf{E}_{1,2}^{(3)} d f_{1} d f_{2} \\
+\int_{\triangle^{2}}\left[S_{\epsilon}\left(f_{1}+f_{2}\right) S_{x}\left(f_{1}\right) S_{x}\left(f_{2}\right)+S_{\epsilon}\left(f_{1}\right) S_{x}\left(f_{2}\right)\right.
\end{gathered}
$$$$
\left.\times S_{x}\left(f_{1}+f_{2}\right)+S_{\epsilon}\left(f_{2}\right) S_{x}\left(f_{1}\right) S_{x}\left(f_{1}+f_{2}\right)\right]
$$$$
\times \mathbf{E}_{1,2}^{(4)} d f_{1} d f_{2}
$$$$
+\int_{\triangle^{2}}\left[S_{\epsilon}\left(f_{1}\right) S_{\epsilon}\left(f_{1}+f_{2}\right) S_{x}\left(f_{2}\right)+S_{\epsilon}\left(f_{2}\right) S_{\epsilon}\left(f_{1}+f_{2}\right)\right.
$$

$$
\left.\times S_{x}\left(f_{1}\right)+S_{\epsilon}\left(f_{1}\right) S_{\epsilon}\left(f_{2}\right) S_{x}\left(f_{1}+f_{2}\right)\right]
$$$$
\times \mathbf{E}_{1,2}^{(4)} d f_{1} d f_{2}
$$$$
+\int_{\triangle^{2}} S_{\epsilon}(0) S_{\epsilon}\left(f_{1}\right) S_{\epsilon}\left(f_{2}\right) \mathbf{E}_{1,2}^{(3)} d f_{1} d f_{2}
$$$$
+\int_{\triangle^{2}} S_{\epsilon}\left(f_{1}\right) S_{\epsilon}\left(f_{2}\right) S_{\epsilon}\left(f_{1}+f_{2}\right) \mathbf{E}_{1,2}^{(4)} d f_{1} d f_{2}
$$

The influence of the additive colored noise on these asymptotic covariances is difficult to analyze from these expressions. However, from the SNR point of view, we note that for a specific distribution of $u_{t}, \mathbf{C}_{2,3}^{x}$ and $\mathbf{C}_{3,3}^{x}$ are proportional to $\sigma_{x}^{5}$ and $\sigma_{x}^{6}$, respectively, whereas the noise additive terms are proportional to $\sigma_{\epsilon}^{2} \sigma_{x}^{3}$ and $\alpha_{1} \sigma_{\epsilon}^{6}+\alpha_{2} \sigma_{\epsilon}^{4} \sigma_{x}^{2}+\alpha_{3} \sigma_{\epsilon}^{2} \sigma_{x}^{4}$, respectively, where the terms $\left(\alpha_{i}\right)_{i=1,2,3}$ depend on the ARMA model and spectral shape of the additive noise. In the case where the noise spectrum has sharp resonances, the dominant term of the previous expression is given by the last term of (4.8). For example, for an AR(1) noise $\left(\epsilon_{t}=e_{t}+b \epsilon_{t-1}\right)$, because it is proved in Appendix $\mathrm{C}$ that

$$
\int_{\triangle^{2}} S_{\epsilon}\left(f_{1}\right) S_{\epsilon}\left(f_{2}\right) S_{\epsilon}\left(f_{1}+f_{2}\right) d f_{1} d f_{2}=\sigma_{\epsilon}^{6}\left(\frac{1+b^{3}}{1-b^{3}}\right)
$$

this dominant term grows unbounded as $b$ approaches +1 and, therefore, contributes to the degradation of the performance when the SNR is decreasing, as will be seen in Section VI-C.

\section{FOURTH-ORDER BARTLETT'S Formula}

In this section, we focus on zero-mean complex processes circular up to the fourth order ${ }^{2}$ as an example.

\section{A. Noise-Free Case}

Under the assumptions of Section II, $\left[\mathbf{c}_{2}^{x}(T), \mathbf{c}_{4}^{x}(T)\right]$ is asymptotically Gaussian ${ }^{3}$ (see, e.g., [1] $)^{4}$

$$
\begin{aligned}
\sqrt{T}\left(\begin{array}{c}
\mathbf{c}_{2}^{x}(T)-\mathbf{c}_{2}^{x} \\
\mathbf{c}_{4}^{x}(T)-\mathbf{c}_{4}^{x}
\end{array}\right) & \stackrel{\mathcal{L}}{\rightarrow} \mathcal{N}_{c}\left(\left(\begin{array}{l}
\mathbf{0} \\
\mathbf{0}
\end{array}\right)\right. \\
& \left.\left(\begin{array}{cc}
\mathbf{C}_{2,2}^{x} & \mathbf{C}_{2,4}^{x} \\
\mathbf{C}_{2,4}^{x} H & \mathbf{C}_{4,4}^{x}
\end{array}\right),\left(\begin{array}{cc}
\mathbf{C}_{2,2}^{\prime x} & \mathbf{C}_{2,4}^{\prime x} \\
\mathbf{C}_{2,4}^{\prime x} T & \mathbf{C}_{4,4}^{\prime x}
\end{array}\right)\right)
\end{aligned}
$$

where $\mathbf{C}_{2,2}^{x}=\lim _{T \rightarrow \infty} T \operatorname{Cov}\left(\mathbf{c}_{2}^{x}(T), \mathbf{c}_{2}^{* x}(T)\right), \mathbf{C}_{2,2}^{\prime x}=$ $\lim _{T \rightarrow \infty} T \operatorname{Cov}\left(\mathbf{c}_{2}^{x}(T), \mathbf{c}_{2}^{x}(T)\right), \quad \mathbf{C}_{2,4}^{x}=\lim _{T \rightarrow \infty} T \operatorname{Cov}$ $\left(\mathbf{c}_{2}^{x}(T), \mathbf{c}_{4}^{* x}(T)\right), \mathbf{C}_{2,4}^{\prime x}=\lim _{T \rightarrow \infty} T \operatorname{Cov}\left(\mathbf{c}_{2}^{x}(T), \mathbf{c}_{4}^{x}(T)\right)$, $\mathbf{C}_{4,4}^{x}=\lim _{T \rightarrow \infty} T \operatorname{Cov}\left(\mathbf{c}_{4}^{x}(T), \mathbf{c}_{4}^{* x}(T)\right)$, and $\mathbf{C}_{4,4}^{\prime x}=$ $\lim _{T \rightarrow \infty} T \operatorname{Cov}\left(\mathbf{c}_{4}^{x}(T), \mathbf{c}_{4}^{x}(T)\right)$. With the approach used to prove the real-valued Bartlett's formula in [13, sec. 4.1, 4.2], it is straightforward to get

$$
\begin{aligned}
\lim _{T \rightarrow \infty} T \operatorname{Cov}\left(c_{k}^{x}(T), c^{* x}(T)\right) & =\sum_{t}\left(c_{t}^{x} c_{-t+k-l}^{x}+c_{k, t+l, t}^{x}\right) \\
\lim _{T \rightarrow \infty} T \operatorname{Cov}\left(c_{k}^{x}(T), c_{l}^{x}(T)\right) & =\sum_{t}\left(c_{t}^{x} c_{-t+k+l}^{x}+c_{k, t, t+l}^{x}\right) .
\end{aligned}
$$

Similarly to the real-valued Bartlett's formula (3.1), we get the following alternative matrix polyspectral Bartlett's formula:

$$
\begin{aligned}
\mathbf{C}_{2,2}^{x}= & \int_{\triangle} S_{x}^{2}(f) \mathbf{e}(f) \mathbf{e}^{H}(f) d f \\
& +\int_{\triangle^{2}} S_{x}\left(f_{1}, f_{2},-f_{2}\right) \mathbf{e}\left(f_{1}\right) \mathbf{e}^{T}\left(f_{2}\right) d f_{1} d f_{2} \\
\mathbf{C}_{2,2}^{\prime x}= & \int_{\triangle} S_{x}^{2}(f) \mathbf{e}(f) \mathbf{e}^{T}(f) d f \\
& +\int_{\triangle^{2}} S_{x}\left(f_{1},-f_{2}, f_{2}\right) \mathbf{e}\left(f_{1}\right) \mathbf{e}^{T}\left(f_{2}\right) d f_{1} d f_{2} .
\end{aligned}
$$

${ }^{2}$ A zero-mean complex processes $x_{t}$ is circular up to the $r$-order iff $\mathrm{E}\left(\prod_{\sum a_{k}=p} x_{t_{k}}^{a_{k}} \prod_{\sum_{b_{l=q}}} x_{t_{l}}^{b_{l}{ }^{*}}\right)=0$ for all positive integers $a_{k}, b_{l}, p, q$ such that $p+q \leq r$ and $p \neq q$.

${ }^{3}$ The distribution of a zero-mean Gaussian complex multivariate random variable $\mathbf{x}$ is characterized by the two covariance matrices $\Sigma_{1} \stackrel{\text { def }}{=} \mathrm{E}\left(\mathbf{x x}^{H}\right)$ and $\Sigma_{2} \stackrel{\text { def }}{=} \mathrm{E}\left(\mathbf{x x}^{T}\right)$. This distribution is denoted $\mathcal{N}\left(\mathbf{0} ; \Sigma_{1}, \Sigma_{2}\right)$.

${ }^{4}$ We note that despite the fact that the cumulants are rich of symmetries, the noncircular complex Gaussian asymptotic distribution of $\mathbf{c}_{2}^{x}(T)$ [resp. $\mathbf{c}_{4}^{x}(T)$ ] is not characterized by $\mathbf{C}_{2,2}^{x}\left[\right.$ resp. $\left.\mathbf{C}_{4,4}^{x}\right]$ only. 
Then, to express $\mathbf{C}_{2,4}^{x}, \mathbf{C}_{2,4}^{x}, \mathbf{C}_{4,4}^{x}, \mathbf{C}_{4,4}^{\prime x}$ in function of the polyspectra of $x_{t}$, we first note that because

$$
\begin{aligned}
& c_{l, m, n}^{x}(T) \stackrel{\text { def }}{=} \frac{1}{T} \sum_{t=1}^{T} x_{t} x_{t+l}^{*} x_{t+m} x_{t+n}^{*}-\left(\frac{1}{T} \sum_{t=1}^{T} x_{t} x_{t+l}^{*}\right) \\
& \times\left(\frac{1}{T} \sum_{t=1}^{T} x_{t+m} x_{t+n}^{*}\right)-\left(\frac{1}{T} \sum_{t=1}^{T} x_{t} x_{t+n}^{*}\right)\left(\frac{1}{T} \sum_{t=1}^{T} x_{t+m} x_{t+l}^{*}\right)
\end{aligned}
$$

we get the following first-order expansion:

$$
\begin{aligned}
c_{l, m, n}^{x}(T)-c_{l, m, n}^{x}=\left(1-c_{n-m}^{x}-c_{l}^{x}-c_{l-m}^{x}-c_{n}^{x}\right) & \underbrace{\left(\begin{array}{c}
\frac{1}{T} \sum_{t} x_{t} x_{t+l}^{*} x_{t+m} x_{t+n}^{*}-\mu_{l, m, n}^{x} \\
\frac{1}{T} \sum_{t} x_{t} x_{t+l}^{*}-c_{l}^{x} \\
\frac{1}{T} \sum_{t} x_{t+m} x_{t+n}^{*}-c_{n-m}^{x} \\
\frac{1}{T} \sum_{t} x_{t} x_{t+n}^{*}-c_{n}^{x} \\
\frac{1}{T} \sum_{t} x_{t+m} x_{t+l}^{*}-c_{l-m}^{x}
\end{array}\right)}_{\boldsymbol{\alpha}_{T}}+\mathrm{o}\left(\boldsymbol{\alpha}_{T}\right)
\end{aligned}
$$

where $\mu_{l, m, n}^{x} \stackrel{\text { def }}{=} \mathrm{E}\left(x_{t} x_{t+l}^{*} x_{t+m} x_{t+n}^{*}\right)$. Using $c_{k}^{x}(T)-c_{k}^{x}=$ $(1 / T) \sum_{t} x_{t} x_{t+k}^{*}-c_{k}^{x}$ and the previous expansion, we get, thanks to the asymptotic covariances of the sample moments derived from [13, rel.10.5.2] extended to the complex case, the asymptotic cross-covariance of the second- and fourth-order sample cumulants

$$
\begin{aligned}
& \lim _{T \rightarrow \infty} T \operatorname{Cov}\left(c_{k}^{x}(T), c_{l, m, n}^{x}(T)\right) \\
& =\left(\begin{array}{lllll}
1 & -c_{n-m}^{x} & -c_{l}^{x} & -c_{l-m}^{x} & -c_{n}^{x}
\end{array}\right) \\
& \times\left(\begin{array}{c}
\sum_{t}\left[\mathrm{E}\left(x_{0} x_{k}^{*} x_{t} x_{t+l}^{*} x_{t+m} x_{t+n}^{*}\right)-c_{k}^{x} \mu_{l, m, n}^{x}\right] \\
\sum_{t}\left[\mathrm{E}\left(x_{0} x_{k}^{*} x_{t} x_{t+l}^{*}\right)-c_{k}^{x} c_{l}^{x}\right] \\
\sum_{t}\left[\mathrm{E}\left(x_{0} x_{k}^{*} x_{t+m} x_{t+n}^{*}\right)-c_{k}^{x} c_{n-m}^{x}\right] \\
\sum_{t}\left[\mathrm{E}\left(x_{0} x_{k}^{*} x_{t} x_{t+n}^{*}\right)-c_{k}^{x} c_{n}^{x}\right] \\
\sum_{t}\left[\mathrm{E}\left(x_{0} x_{k}^{*} x_{t+m} x_{t+l}^{*}\right)-c_{k}^{x} c_{l-m}^{x}\right]
\end{array}\right) .
\end{aligned}
$$

To get $\lim _{T \rightarrow \infty} T \operatorname{Cov}\left(c_{k, l, m}^{x}(T), c_{n, p, q}^{x}(T)\right)$, we use the same approach for which we have

$$
\begin{aligned}
\lim _{T \rightarrow \infty} & T \operatorname{Cov}\left(c_{k, l, m}^{x}(T), c_{n, p, q}^{x}(T)\right) \\
= & \left(1,-c_{m-l}^{x},-c_{k}^{x},-c_{k-l}^{x},-c_{m}^{x}\right)\left(\begin{array}{cc}
c_{1} & \mathbf{c}_{3}^{T} \\
\mathbf{c}_{2} & \mathbf{C}
\end{array}\right) \\
& \times\left(1,-c_{q-p}^{x},-c_{n}^{x},-c_{n-p}^{x},-c_{q}^{x}\right)^{T}
\end{aligned}
$$

with

$$
\begin{aligned}
& c_{1}=\sum_{t}\left[\mathrm{E}\left(x_{0} x_{k}^{*} x_{l} x_{m}^{*} x_{t} x_{t+n}^{*} x_{t+p} x_{t+q}^{*}\right)-\mu_{k, l, m}^{x} \mu_{n, p, q}^{x}\right] \\
& \mathbf{c}_{2}=\left[\begin{array}{c}
\sum_{t}\left[\mathrm{E}\left(x_{0} x_{k}^{*} x_{t} x_{t+n}^{*} x_{t+p} x_{t+q}^{*}\right)-c_{k}^{x} \mu_{n, p, q}^{x}\right] \\
\sum_{t}\left[\mathrm{E}\left(x_{l} x_{m}^{*} x_{t} x_{t+n}^{*} x_{t+p} x_{t+q}^{*}\right)-c_{m-l}^{x} \mu_{n, p, q}^{x}\right] \\
\sum_{t}\left[\mathrm{E}\left(x_{0} x_{m}^{*} x_{t} x_{t+n}^{*} x_{t+p} x_{t+q}^{*}\right)-c_{m}^{x} \mu_{n, p, q}^{x}\right] \\
\sum_{t}\left[\mathrm{E}\left(x_{l} x_{k}^{*} x_{t} x_{t+n}^{*} x_{t+p} x_{t+q}^{*}\right)-c_{k-l}^{x} \mu_{n, p, q}^{x}\right]
\end{array}\right] \\
& \mathbf{c}_{3}=\left[\begin{array}{c}
\sum_{t}\left[\mathrm{E}\left(x_{0} x_{n}^{*} x_{t} x_{t+k}^{*} x_{t+l} x_{t+m}^{*}\right)-c_{n}^{x} \mu_{k, l, m}^{x}\right] \\
\sum_{t}\left[\mathrm{E}\left(x_{p} x_{q}^{*} x_{t} x_{t+k}^{*} x_{t+l} x_{t+m}^{*}\right)-c_{q-p}^{x} \mu_{k, l, m}^{x}\right] \\
\sum_{t}\left[\mathrm{E}\left(x_{0} x_{q}^{*} x_{t} x_{t+k}^{*} x_{t+l} x_{t+m}^{*}\right)-c_{q}^{x} \mu_{k, l, m}^{x}\right] \\
\sum_{t}\left[\mathrm{E}\left(x_{p} x_{n}^{*} x_{t} x_{t+k}^{*} x_{t+l} x_{t+m}^{*}\right)-c_{n-p}^{x} \mu_{k, l, m}^{x}\right]
\end{array}\right]
\end{aligned}
$$

where we have the equation at the bottom of the page for the continuation of the above. To proceed, the moments in the last four summations of (5.3) and in $\mathbf{C}$ are expressed by cumulants. For example

$$
\begin{aligned}
& \sum_{t}\left[\mathrm{E}\left(x_{0} x_{k}^{*} x_{t} x_{t+l}^{*}\right)-c_{k}^{x} c_{l}^{x}\right] \\
& =\sum_{t}\left(c_{t}^{x} c_{-t+k+l}^{x}+c_{k, t, t+l}^{x}\right) \\
& \sum_{t}\left[\mathrm{E}\left(x_{0} x_{k}^{*} x_{t+m} x_{t+n}^{*}\right)-c_{k}^{x} c_{n-m}^{x}\right] \\
& =\sum_{t}\left(c_{t+n}^{x} c_{-t+k-m}^{x}+c_{k, t+m, t+n}^{x}\right) \\
& \sum_{t}\left[\mathrm{E}\left(x_{0} x_{k}^{*} x_{t} x_{t+n}^{*}\right)-c_{k}^{x} c_{n}^{x}\right] \\
& =\sum_{t}\left(c_{t}^{x} c_{-t+k+n}^{x}+c_{k, t, t+n}^{x}\right) \\
& \sum_{t}\left[\mathrm{E}\left(x_{0} x_{k}^{*} x_{t+m} x_{t+l}^{*}\right)-c_{k}^{x} c_{l-m}^{x}\right] \\
& =\sum_{t}\left(c_{t+l}^{x} c_{-t+k-m}^{x}+c_{k, t+m, t+l}^{x}\right)
\end{aligned}
$$

then, these summations are evaluated similarly to the real-valued polyspectral Bartlett's formula (3.1).

The summations of sixth-order moments in $\mathbf{c}_{2}, \mathbf{c}_{3}$, and in the first term of (5.3) and summations of eighth-order moments in $c_{1}$ are expressed as functions of polyspectra of $x_{t}$ from our symbolic calculus akin to a high-level language based on a few well-defined rules used in Section IV. Here, the zero
$(\mathbf{C})_{1,1}=\left[\begin{array}{c}\sum_{t}\left[\mathrm{E}\left(x_{0} x_{k}^{*} x_{t} x_{t+n}^{*}\right)-c_{k}^{x} c_{n}^{x}\right] \\ \sum_{t}\left[\mathrm{E}\left(x_{l} x_{m}^{*} x_{t} x_{t+n}^{*}\right)-c_{m-l}^{x} c_{n}^{x}\right]\end{array}\right.$
$\sum_{t}\left[\mathrm{E}\left(x_{0} x_{k}^{*} x_{t+p} x_{t+q}^{*}\right)-c_{k}^{x} c_{q-p}^{x}\right]$
$\sum_{t}\left[\mathrm{E}\left(x_{l} x_{m}^{*} x_{t+p} x_{t+q}^{*}\right)-c_{m-l}^{x} c_{q-p}^{x}\right]$
$(\mathbf{C})_{2,1}=\left[\begin{array}{l}\sum_{t}\left[\mathrm{E}\left(x_{0} x_{m}^{*} x_{t} x_{t+n}^{*}\right)-c_{m}^{x} c_{n}^{x}\right] \\ \sum_{t}\left[\mathrm{E}\left(x_{l} x_{k}^{*} x_{t} x_{t+n}^{*}\right)-c_{k-l}^{x} c_{n}^{x}\right]\end{array}\right.$
$\left.\sum_{t}\left[\mathrm{E}\left(x_{0} x_{m}^{*} x_{t+p} x_{t+q}^{*}\right)-c_{m}^{x} c_{q-p}^{x}\right]\right]$
$(\mathbf{C})_{1,2}=\left[\begin{array}{c}\sum_{t}\left[\mathrm{E}\left(x_{0} x_{k}^{*} x_{t} x_{t+q}^{*}\right)-c_{k}^{x} c_{q}^{x}\right] \\ \sum_{t}\left[\mathrm{E}\left(x_{l} x_{m}^{*} x_{t} x_{t+q}^{*}\right)-c_{m-l}^{x} c_{q}^{x}\right]\end{array}\right.$
$\left.\sum_{t}\left[\mathrm{E}\left(x_{l} x_{k}^{*} x_{t+p} x_{t+q}^{*}\right)-c_{k-l}^{x} c_{q-p}^{x}\right]\right]$
$(\mathbf{C})_{2,2}=\left[\begin{array}{l}\sum_{t}\left[\mathrm{E}\left(x_{0} x_{m}^{*} x_{t} x_{t+q}^{*}\right)-c_{m}^{x} c_{q}^{x}\right] \\ \sum_{t}\left[\mathrm{E}\left(x_{l} x_{k}^{*} x_{t} x_{t+q}^{*}\right)-c_{k-l}^{x} c_{q}^{x}\right]\end{array}\right.$
$\sum_{t}\left[\mathrm{E}\left(x_{0} x_{k}^{*} x_{t+p} x_{t+n}^{*}\right)-c_{k}^{x} c_{n-p}^{x}\right]$
$\left.\sum_{t}\left[\mathrm{E}\left(x_{l} x_{m}^{*} x_{t+p} x_{t+n}^{*}\right)-c_{m-l}^{x} c_{n-p}^{x}\right]\right]$
$\left.\begin{array}{l}\sum_{t}\left[\mathrm{E}\left(x_{0} x_{m}^{*} x_{t+p} x_{t+n}^{*}\right)-c_{m}^{x} c_{n-p}^{x}\right] \\ \sum_{t}\left[\mathrm{E}\left(x_{l} x_{k}^{*} x_{t+p} x_{t+n}^{*}\right)-c_{k-l}^{x} c_{n-p}^{x}\right]\end{array}\right]$. 
terms are eliminated according to the circularity property up to the fourth order, and the sets of similar expressions are constructed w.r.t. the number of terms $x_{t+a}$ and $x_{t+b}^{*}$ in each product of cumulants. For each such set of similar expressions [six sets for $\mathrm{E}\left(x_{0} x_{k}^{*} x_{t} x_{t+l}^{*} x_{t+m} x_{t+n}^{*}\right)$ and 21 sets for $\left.\mathrm{E}\left(x_{0} x_{k}^{*} x_{l} x_{m}^{*} x_{t} x_{t+n}^{*} x_{t+p} x_{t+q}^{*}\right)\right]$, a representative term is chosen to be analytically expressed by a polyspectral formula derived in the same way as for the third-order case proved in Appendix A. We get

$$
\begin{aligned}
\mathrm{E}\left(x_{0}\right. & \left.x_{k}^{*} x_{t} x_{t+l}^{*} x_{t+m} x_{t+n}^{*}\right) \\
= & \operatorname{Cum}\left(x_{0}, x_{k}^{*}, x_{t}, x_{t+l}^{*}, x_{t+m}, x_{t+n}^{*}\right) \\
& +\operatorname{Cum}\left(x_{0}, x_{k}^{*}, x_{t}, x_{t+l}^{*}\right) \operatorname{Cum}\left(x_{t+m}, x_{t+n}^{*}\right) \\
& +\operatorname{Cum}\left(x_{0}, x_{k}^{*}\right) \operatorname{Cum}\left(x_{t}, x_{t+l}^{*}\right) \\
& \times \operatorname{Cum}\left(x_{t+m}, x_{t+n}^{*}\right)(6) .
\end{aligned}
$$

and

$$
\begin{aligned}
\mathrm{E}\left(x_{0}\right. & \left.x_{k}^{*} x_{l} x_{m}^{*} x_{t} x_{t+n}^{*} x_{t+p} x_{t+q}^{*}\right) \\
= & \operatorname{Cum}\left(x_{0}, x_{k}^{*}, x_{l}, x_{m}^{*}, x_{t}, x_{t+n}^{*}, x_{t+p}, x_{t+q}^{*}\right) \\
& +\operatorname{Cum}\left(x_{0}, x_{k}^{*}, x_{l}, x_{m}^{*}, x_{t}, x_{t+n}^{*}\right) \operatorname{Cum}\left(x_{t+p}, x_{t+q}^{*}\right) \\
& +\operatorname{Cum}\left(x_{0}, x_{k}^{*}, x_{l}, x_{m}^{*}\right) \operatorname{Cum}\left(x_{t}, x_{t+n}^{*}, x_{t+p}, x_{t+q}^{*}\right) \\
& +\operatorname{Cum}\left(x_{0}, x_{k}^{*}, x_{l}, x_{m}^{*}\right) \operatorname{Cum}\left(x_{t}, x_{t+n}^{*}\right) \\
& \times \operatorname{Cum}\left(x_{t+p}, x_{t+q}^{*}\right)(72) \\
& +\operatorname{Cum}\left(x_{0}, x_{k}^{*}\right) \operatorname{Cum}\left(x_{l}, x_{m}^{*}\right) \operatorname{Cum}\left(x_{t}, x_{t+n}^{*}\right) \\
& \times \operatorname{Cum}\left(x_{t+p}, x_{t+q}^{*}\right)(24)
\end{aligned}
$$

where, for example, the second line of (5.4) gives

$$
\begin{aligned}
\operatorname{Cum} & \left(x_{0}, x_{k}^{*}, x_{t}, x_{t+l}^{*}\right) \operatorname{Cum}\left(x_{t+m}, x_{t+n}^{*}\right)(9) \\
= & c_{k, t, t+l}^{x} c_{n-m}^{x}+c_{k, t, t+n}^{x} c_{l-m}^{x}+c_{k, t+m, t+l}^{x} c_{n}^{x} \\
& +c_{k, t+m, t+n}^{x} c_{l}^{x}+c_{l,-t, n}^{x} c_{t+m-k}^{x}+c_{l-m,-t-m, n-m}^{x} c_{t-k}^{x} \\
& +c_{n, m,-t+k}^{x} c_{t+l}^{x}+c_{l, m,-t+k}^{x} c_{t+n}^{x}+c_{k}^{x} c_{l, m, n}^{x}
\end{aligned}
$$

and because the last term cancels with $-c_{k}^{x} \mu_{l, m, n}^{x}$ in the summation $\sum_{t}\left[\mathrm{E}\left(x_{0} x_{k}^{*} x_{t} x_{t+l}^{*} x_{t+m} x_{t+n}^{*}\right)-c_{k}^{x} \mu_{l, m, n}^{x}\right]$, this line gives three sets of similar expressions.

The limits $\quad \lim _{T \rightarrow \infty} T \operatorname{Cov}\left(c_{k}^{x}(T), c_{l, m, n}^{* x}(T)\right) \quad$ and $\lim _{T \rightarrow \infty} T \operatorname{Cov}\left(c_{k, l, m}^{x}(T), c_{n, p, q}^{* x}(T)\right)$ are evaluated similarly. Finally, our symbolic calculus delivers

- LATEX polyspectral expressions of $\mathbf{C}_{2,4}^{x}, \mathbf{C}_{2,4}^{\prime x}, \mathbf{C}_{4,4}^{x}$, and $\mathbf{C}_{4,4}^{\prime x}$ similar to (4.5), (4.6), but not reproduced here due to lack of space. They are available from the authors upon request;

- Matlab function files allowing one to compute the numerical values of these expressions (see Section VI-B).

We have chosen to consider zero-mean processes that are complex circular up to the fourth order. Naturally, our methodology can be applied to the cases of zero-mean real-valued processes or zero-mean complex processes circular up to the second order. The only difference is due to distinct rules of elimination of the zero terms.

\section{B. Noisy Case}

In the noisy case, the fourth-order relations are derived in the same way as for the second and third order, thanks to the additivity of the polyspectra of $x_{t}$ and $\epsilon_{t}$.

\section{APPLICATION to ESTIMATION OF ARMA PARAMETERS}

It is beyond the scope of this paper to analyze the statistical performance of the identification algorithms based on sample third- or fourth-order cumulants proposed in the literature. Instead, we unveil the influence of colored additive noise on the potential asymptotic performance of such an arbitrary algorithm. In that purpose, asymptotic lower bound for the covariance of third- or fourth-order estimators and asymptotic covariance of an arbitrary third- or fourth-order-based algorithm are considered, where special attention is given to the statistics involved.

\section{A. Asymptotic Lower Bound on the Covariance}

To apply the notion of asymptotic minimum variance (AMV) estimators [5] (also called asymptotically best consistent estimators in [15]), the involved sample cumulants $\mathbf{c}^{y}(T)$ must satisfy three conditions.

- If $\Theta$ denotes the real-valued parameters (real and imaginary parts in the case of complex processes) of the noisy ARMA model, $\Theta$ must be identifiable from $\mathbf{c}^{y}(\Theta)$ in the following sense: $\mathbf{c}^{y}(\Theta)=\mathbf{c}^{y}\left(\Theta^{\prime}\right) \Rightarrow \Theta=\Theta^{\prime} .5$

- The involved third- or fourth-order algorithms considered as mappings that associate to $\mathbf{c}^{y}(T)$, the estimate $\Theta(T)$ : $\mathbf{c}^{y}(T) \longmapsto \Theta(T)=\operatorname{alg}\left(\mathbf{c}^{y}(T)\right)$ must be real [resp. complex] differentiable w.r.t. $\mathbf{c}^{y}(T)$ at the point $\mathbf{c}^{y}(\Theta)$ for real-[resp. complex] valued processes.

- The covariance $\boldsymbol{\Sigma}$ of the asymptotic distribution of the sample cumulants $\mathbf{c}^{y}(T)$ must be nonsingular.

These two latter conditions do not raise any problem for real-valued processes. However, for complex-valued processes, $\mathbf{c}^{y}(T)$ must collect real-valued cumulants [e.g., $c_{0}^{y}(T)$ and $\left.c_{0,0,0}^{y}(T)\right]$ and complex-valued cumulants and their conjugate [e.g., $c_{k}^{y}(T)$ and $c_{k}^{y *}(T)$ for $k \neq 0$ ] to satisfy the second condition (see [16]). In addition, to satisfy the third condition, redundant cumulant samples must be withdrawn. In these conditions, the asymptotic covariance $\mathbf{C}_{\Theta}$ of an estimator of $\Theta$ given by an arbitrary third- or fourth-order algorithm is bounded below by the real symmetric positive definite matrix $\left[\mathbf{F}^{H}(\Theta) \boldsymbol{\Sigma}^{-1}(\Theta) \mathbf{F}(\Theta)\right]^{-1}$

$$
\mathbf{C}_{\Theta} \geq\left[\mathbf{F}^{H}(\Theta) \boldsymbol{\Sigma}^{-1}(\Theta) \mathbf{F}(\Theta)\right]^{-1}
$$

where $\mathbf{F}(\Theta) \stackrel{\text { def }}{=}\left(d \mathbf{c}^{y}(\Theta) / d \Theta\right)$. Furthermore, there exists a nonlinear least-square algorithm (dubbed the AMV algorithm [5]) whose covariance of the asymptotic distribution of the estimate of $\Theta$ satisfies (6.1) with equality. In practice, $\epsilon_{t}$ is Gaussian distributed, and if third- or fourth-order cumulants are considered, the parametrization $\Theta$ can be partitioned as $\Theta=\left[\Theta_{1}^{T}, \Theta_{2}^{T}\right]^{T}$, where $\Theta_{1}$ collects the parameters of the ARMA filter of interest, and $\Theta_{2}$ collects the parameters $\sigma_{u}^{2}, \kappa_{3_{u}}, \ldots, \sigma_{e}^{2}$ of the sequences $u_{t}$ and $e_{t}$. Consequently, the covariance of the asymptotic distribution of the minimum variance third- or fourth-order ARMA estimator is given by the top left "ARMA corner" of $\left[\mathbf{F}^{H}(\Theta) \Sigma^{-1}(\Theta) \mathbf{F}(\Theta)\right]^{-1}$. Then, because $\mathbf{c}^{y}(\Theta)$ is linear with

\footnotetext{
${ }^{5}$ We note that the definition of $\Theta$ depends on the choice of the cumulants $c^{y}$ and the a priori knowledge on the distribution of the measurement noise $\epsilon_{t}$.
} 
respect to $\Theta_{2}$, i.e., $\mathbf{c}^{y}(\Theta)=\bar{\Psi}\left(\Theta_{1}\right) \Theta_{2}$ implies $\mathbf{F}=\left[\mathbf{F}_{1}, \mathbf{\Psi}\right]$ with $\mathbf{F}_{1} \stackrel{\text { def }}{=}\left(\partial \mathbf{c}^{y}(\Theta) / \partial \Theta_{1}\right)$, the matrix inversion lemma gives

$$
\begin{aligned}
\mathbf{C}_{\Theta_{1}} & =\left(\mathbf{F}_{1}^{H} \boldsymbol{\Sigma}^{-1} \mathbf{F}_{1}-\mathbf{F}_{1}^{H} \boldsymbol{\Sigma}^{-1} \Psi\left[\Psi^{H} \boldsymbol{\Sigma}^{-1} \mathbf{\Psi}\right]^{-1} \Psi^{H} \boldsymbol{\Sigma}^{-1} \mathbf{F}_{1}\right)^{-1} \\
& =\left(\mathbf{F}_{1}^{T} \boldsymbol{\Sigma}^{-\frac{1}{2}} \mathbf{P}_{\boldsymbol{\Sigma}^{-\frac{1}{2}}}^{\perp} \boldsymbol{\Sigma}^{-\frac{1}{2}} \mathbf{F}_{1}\right)^{-1}
\end{aligned}
$$

where $\mathbf{P}_{\Sigma-1 / 2}^{\perp}$ denotes the projector onto the orthogonal complement of the columns of $\Sigma^{-1 / 2} \Psi$.

\section{B. Asymptotic Covariance of an Arbitrary Third-or Fourth-Order Estimator}

The asymptotic performance of an arbitrary third- or fourth-order-based algorithm that estimates the ARMA parameters $\Theta_{1}$ of a noisy ARMA model can be derived (see, e.g., [13, Th. 3.16]) from the asymptotic normality of $\left[\mathbf{c}_{2}^{y}(T), \mathbf{c}_{3}^{y}(T)\right]$ or $\left[\mathbf{c}_{2}^{y}(T), \mathbf{c}_{4}^{y}(T)\right]$

$$
\sqrt{T}\left(\Theta_{1}(T)-\Theta_{1}\right) \stackrel{\mathcal{L}}{\rightarrow} \mathcal{N}\left(\mathbf{0} ; \mathbf{G}(\Theta) \mathbf{\Sigma}(\Theta) \mathbf{G}^{H}(\Theta)\right)
$$

where $\mathbf{G}(\Theta)$ is the differential of the algorithm considered as a mapping, evaluated at point $\Theta$, and $\Sigma(\Theta)$ is the asymptotic covariance matrix of the sample cumulants involved in the algorithm deduced from $\mathbf{C}_{2,2}^{y}, \mathbf{C}_{2,3}^{y}, \mathbf{C}_{3,3}^{y}$ for the third-order real case or $\mathbf{C}_{2,2}^{y}, \mathbf{C}_{2,2}^{\prime y}, \mathbf{C}_{2,4}^{y}, \mathbf{C}_{2,4}^{\prime y}, \mathbf{C}_{4,4}^{y}, \mathbf{C}_{4,4}^{\prime y}$ for the fourth-order complex case.

Our symbolic calculus translates the polyspectral expressions of these different asymptotic covariance matrices into rational fraction expressions w.r.t. the ARMA process $x_{t}$ and $\operatorname{AR}(1)$ process $\epsilon_{t}$ coefficients, under the form of Matlab function files. These files allow one to compute the numerical values of these matrices for particular values of the parameters and are available from the authors upon request. They allow the interested practitioner to evaluate the performance of third- or fourth-order algorithms by simple computation of the differential $\mathbf{G}(\Theta)$ of the algorithm and selection of the $\boldsymbol{\Sigma}(\Theta)$ involved. The programs giving the numerical values of $\left(\mathbf{C}_{i, j}^{y}\right)_{i, j=2,3}$ and $\left(\mathbf{C}_{i, j}^{y}, \mathbf{C}_{i, j}^{\prime}\right)_{i, j=2,4}$ are built along the following steps. First, each polyspectral integral expression obtained in Section IV and V is symbolically expressed as functions of $H(f) \stackrel{\text { def }}{=} \sum_{n} h_{n} e^{-i 2 \pi n f}$ and of the transfer function $G(f)$ of the measurement noise generator driven by the independent Gaussian sequence $e_{t}$ of power $\sigma_{e}^{2}$, thanks to the relations (see, e.g., [4, rel. (C-24)]) extended to the complex case

$$
\begin{aligned}
S_{y}(f)= & \sigma_{u}^{2} H^{*}(-f) H(-f)+\sigma_{e}^{2} G^{*}(-f) G(-f) \\
S_{y}\left(f_{1}, \ldots, f_{p}\right)= & \kappa_{p+1_{u}} H\left(f_{1}\right) H\left(f_{2}\right) \ldots H\left(f_{p}\right) \\
& \times H\left(-f_{1}-f_{2} \ldots-f_{p-1}\right) \\
& p>1(\text { real case }) \\
S_{y}\left(f_{1}, \ldots, f_{2 p-1}\right)= & \kappa_{2 p_{u}} H^{*}\left(-f_{1}\right) H\left(f_{2}\right) \ldots H^{*}\left(-f_{2 p-1}\right) \\
& \times H\left(-f_{1}-f_{2} \ldots-f_{2 p-1}\right) \\
& p>1(\text { complex case }) .
\end{aligned}
$$

Second, the transfer functions $H(f)$ and $G(f)$ are expressed in terms of the parameters of the MA process $x_{t}$ and AR process $\epsilon_{t}$ (e.g., $a$ and $b$ in Section VI-C), and finally, the polyspectral

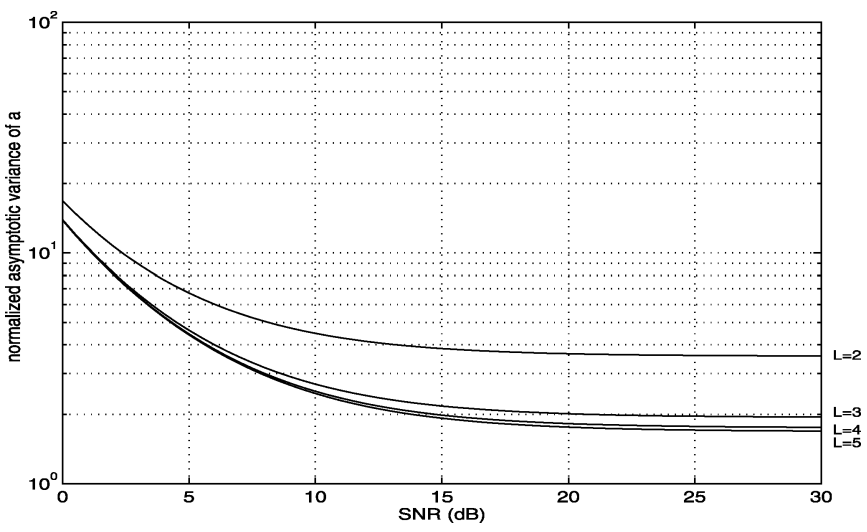

Fig. 1. Third-order normalized lower bound for the asymptotic variance of estimates of $a$ as a function of the SNR for different values of $L$.

integrals are symbolically computed as functions of the MA and AR parameters for $\left(L_{i}\right)_{i=1, \ldots, 4}$ fixed, thanks to the relation

$$
\int_{\triangle^{k}} \sum_{\alpha_{1}} \ldots \sum_{\alpha_{k}} c_{\alpha_{1} \ldots \alpha_{k}} e^{-i 2 \pi\left(\alpha_{1} f_{1}+\ldots+\alpha_{k} f_{k}\right)} \times
$$

$$
d f_{1} d f_{2} \ldots d f_{k}=c_{0 \ldots 0}
$$

where $\sum_{\alpha_{1}} \ldots \sum_{\alpha_{k}} c_{\alpha_{1} \ldots \alpha_{k}} e^{-i 2 \pi\left(\alpha_{1} f_{1}+\ldots+\alpha_{k} f_{k}\right)}$ are deduced from the polyspectral expressions evaluated as functions of $H(f)$ and $G(f)$.

\section{Illustrative Numerical Examples}

As examples, three experiments are proposed for which noisy $\mathrm{MA}(1)$ or $\mathrm{MA}(2)$ processes are considered. The SNR is defined as $S N R(d B)=10 \log _{10}\left(\mathrm{E}\left(x_{t}^{2}\right) / \mathrm{E}\left(\epsilon_{t}^{2}\right)\right)$. In the two first experiments, the processes are real valued, the input $u_{t}$ is exponentially distributed with mean adjusted to zero, power $\sigma_{u}^{2}$ and $\kappa_{k_{u}}=(k-1) ! \sigma_{u}^{k}$, and the measurement noise $\epsilon_{t}$ is either Gaussian i.i.d. or Gaussian $\operatorname{AR}(1),\left(\epsilon_{t}=e_{t}+b \epsilon_{t-1}\right.$ where $e_{t}$ is Gaussian i.i.d.).

In the first experiment, an MA(1) is considered, where $\Theta_{1}=$ $a$, and $\Theta_{2}=\kappa_{3_{u}}$. Fig. 1 shows the normalized asymptotic lower bound $^{6}$ for the asymptotic variance of estimates of $a$ based on the third-order diagonal ${ }^{7}$ cumulants $\left\{c_{k, k}^{y}(T) ; k=0, \ldots, L-1\right\}$ and $\left\{c_{0, k}^{y}(T) ; k=1, \ldots, L-1\right\}^{8}$ as a function of the SNR for different values of $L$ for white noise. For $k \geq 2$, the sample cumulants $c_{k, k}^{y}(T)$ and $c_{0, k}^{y}(T)$ are consistent estimates of zero. Nevertheless, however, Fig. 1 shows that they contribute to improving the performance. This extends to noisy processes an observation shown in the noise-free case in [5].

Figs. 2 and 3 show the lower bound for the asymptotic variance of estimates of $a(a=0.5)$ based on the sample third-order cumulants $\left(c_{0,0}^{y}(T), c_{0,1}^{y}(T), c_{1,1}^{y}(T)\right)$ and the asymptotic theoretical and empirical variance given by the so-called $C(k, q)$

\footnotetext{
${ }^{6}$ The normalized asymptotic lower bounds and asymptotic theoretical variances are computed for $T=1$. That means that the actual asymptotic lower bounds and asymptotic theoretical variances are obtained from the results given here by dividing by $T$.

${ }^{7}$ We restrict this example to diagonal cumulants because the off-diagonal third-order cumulants carry almost no additional information beyond the information in the diagonal ones in the MA(1) process case, as it was shown in the noise-free case in [5].

${ }^{8}$ The cumulants in the second set are called diagonal because of the relation$\operatorname{ship} c_{0, k}^{y}=c_{-k,-k}^{y}$.
} 


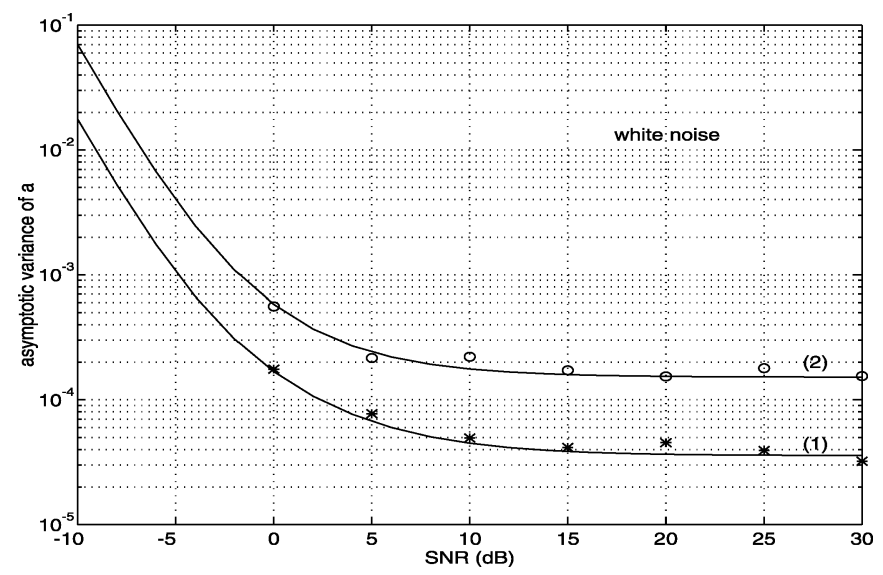

Fig. 2. Third-order asymptotic lower bound (1) and asymptotic theoretical and empirical (averaged on 100 independent Monte Carlo runs) variance given by the $C(k, q)$ formula (2) as a function of the SNR for $T=10^{5}$.

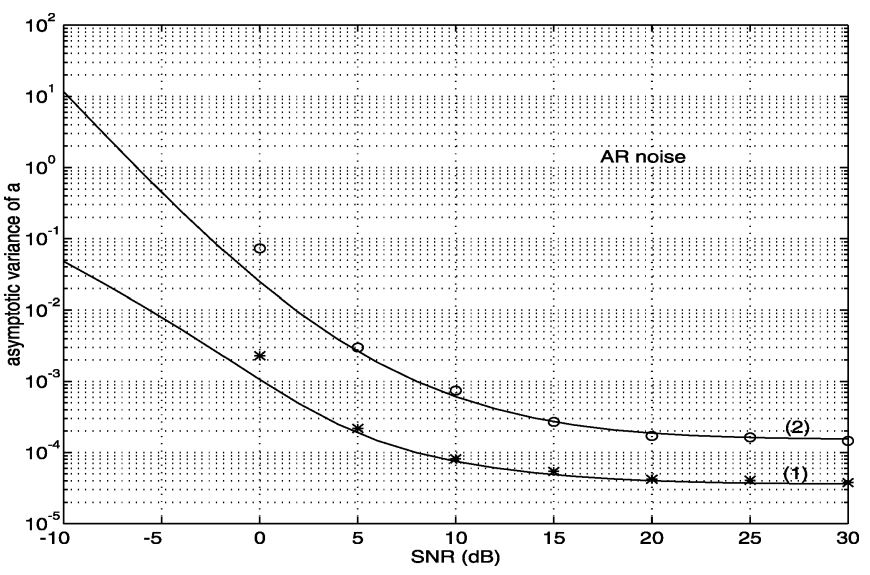

Fig. 3. Third-order asymptotic lower bound (1) and asymptotic theoretical and empirical (averaged on 100 independent Monte Carlo runs) variance given by the $C(k, q)$ formula (2) as a function of the SNR for $T=10^{5}$.

formula of Giannakis [3] $\left(a(T)=c_{1,1}^{y}(T) / c_{0,1}^{y}(T)\right)$, respectively, for white and $\operatorname{AR}(1)$ noise $(b=0.99)$ as a function of the SNR. Because $\mathbf{G}(\Theta)=\left[-\left(1 / \kappa_{3_{u}}\right),\left(1 / a \kappa_{3_{u}}\right)\right]=((1+$ $\left.\left.a^{2}\right)^{3 / 2} / 2 \sigma_{x}^{3}\right)[-1,(1 / a)]$, we note that from the expression of $\mathbf{C}_{3,3}^{y}$ given in Section IV-B, we get for the $C(k, q)$ estimator

$$
\lim _{T \rightarrow \infty} T \operatorname{Var}(a(T))=v_{0}+\left[\beta_{1}\left(\frac{\sigma_{\epsilon}^{2}}{\sigma_{x}^{2}}\right)+\beta_{2}\left(\frac{\sigma_{\epsilon}^{4}}{\sigma_{x}^{4}}\right)+\beta_{3}\left(\frac{\sigma_{\epsilon}^{6}}{\sigma_{x}^{6}}\right)\right]
$$

where $v_{0}$ does not depend on the powers of $x_{t}$ and $\epsilon_{t}$. We see from these two figures that below a certain threshold (which is typically related to the noise spectrum), the asymptotic variance of the estimate of $a$ grows rapidly with the noise level. Beyond this threshold (for SNR > $10 \mathrm{~dB}$ [resp. SNR > $20 \mathrm{~dB}$ ] for white noise [resp. AR(1) noise], the asymptotic variance is approximately constant. This proves that the AMV3 and the $C(k, q)$ algorithms are insensitive to noise in a large domain. Furthermore, we note that contrary to the asymptotic lower bound, the asymptotic variance given by the $C(k, q)$ algorithm strongly degrades for sharp resonant AR(1) noise compared to white noise of the same power.

Figs. 4 and 5 show the asymptotic lower bound and the asymptotic theoretical and empirical variance given by the

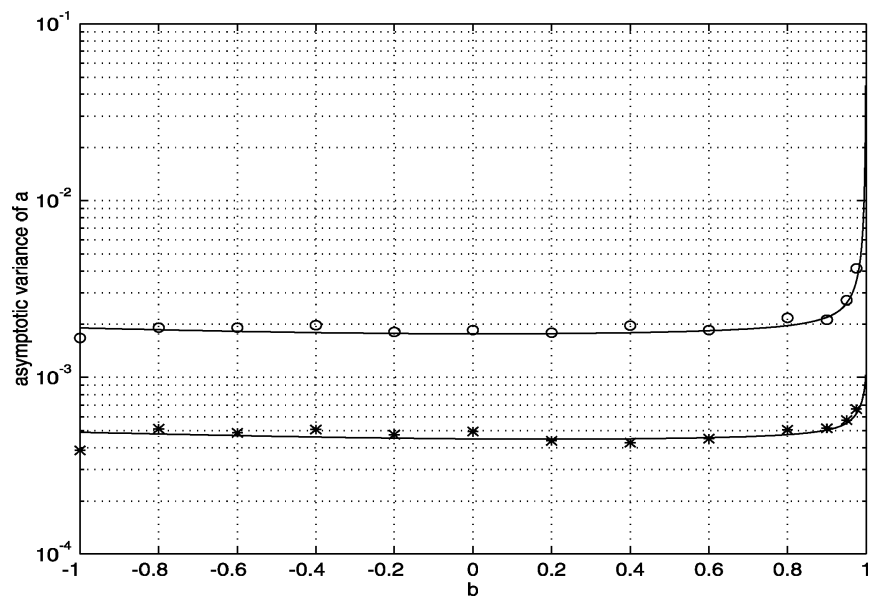

Fig. 4. Third-order asymptotic lower bound (1) and asymptotic theoretical and empirical (averaged on 1000 independent Monte Carlo runs) variance given by the $C(k, q)$ formula (2) as a function of the noise parameter $b$ for $a=0.5$, $\mathrm{SNR}=10 \mathrm{~dB}$, and $T=10^{4}$.

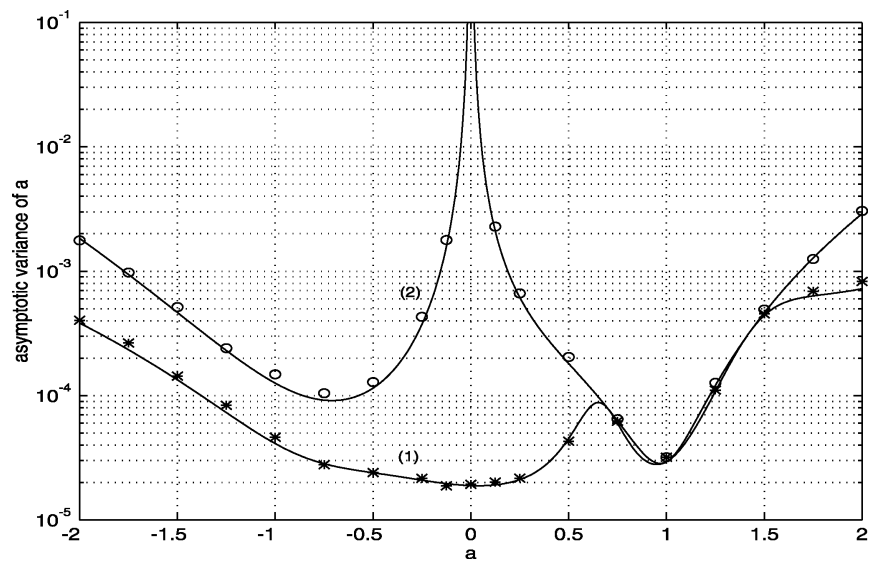

Fig. 5. Third-order asymptotic lower bound (1) and asymptotic theoretical and empirical (averaged on 1000 independent Monte Carlo runs) variance given by the $C(k, q)$ formula (2) as a function of the MA parameter $a$ for $b=0.5$, $\mathrm{SNR}=10 \mathrm{~dB}$, and $T=10^{4}$.

$C(k, q)$ algorithm as a function of the parameter $b$ of the $\mathrm{AR}(1)$ noise $\epsilon_{t}$ and of the parameter $a$ of the MA(1) process $x_{t}$, respectively. We see that the performance is very sensitive to the spectrum of the MA(1) process $x_{t}$ but relatively insensitive to the spectrum of the additive noise $\epsilon_{t}$, except when $b$ approaches 1 [see (4.9)], where the performance of any third order-based algorithm dramatically degrades. We note that the asymptotic variance given by the $C(k, q)$ algorithm attains the asymptotic lower bound for $a=1^{9}$ and is inadequate to estimate a parameter $a$ close to zero.

Figs. 6(a) and (b) show the theoretical asymptotic lower bound and the empirical asymptotic variance given by the AMV3 and the $C(k, q)$ algorithms as a function of the number $T$ of samples for two values of SNR. We see that the domain of validity of our asymptotic analysis roughly does not depend on the algorithm but is sensitive to the SNR. Naturally, this domain of validity increases with increasing SNR $(T>4000$ for $S N R=0 \mathrm{~dB}$ and $T>1000$ for $S N R=20 \mathrm{~dB}$ ).

${ }^{9}$ This property has been confirmed for all values of $b$ and SNR, but we have not succeeded in proving it analytically. 


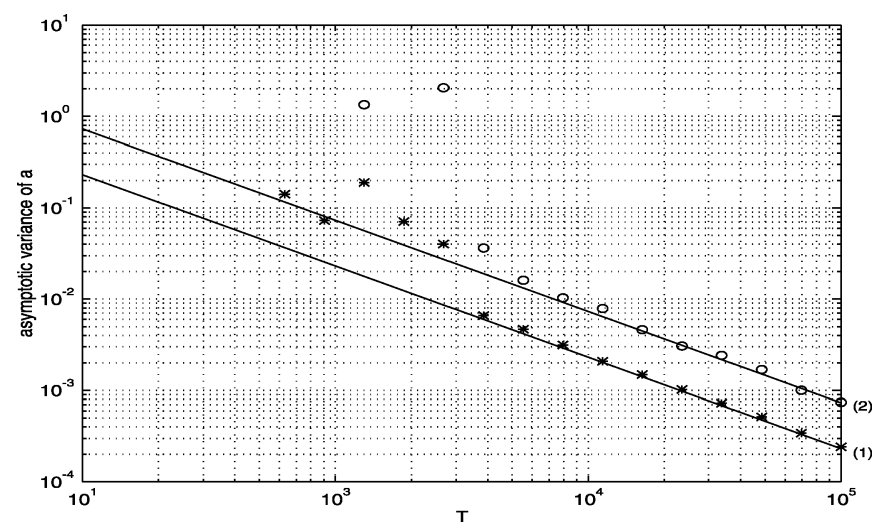

(a)

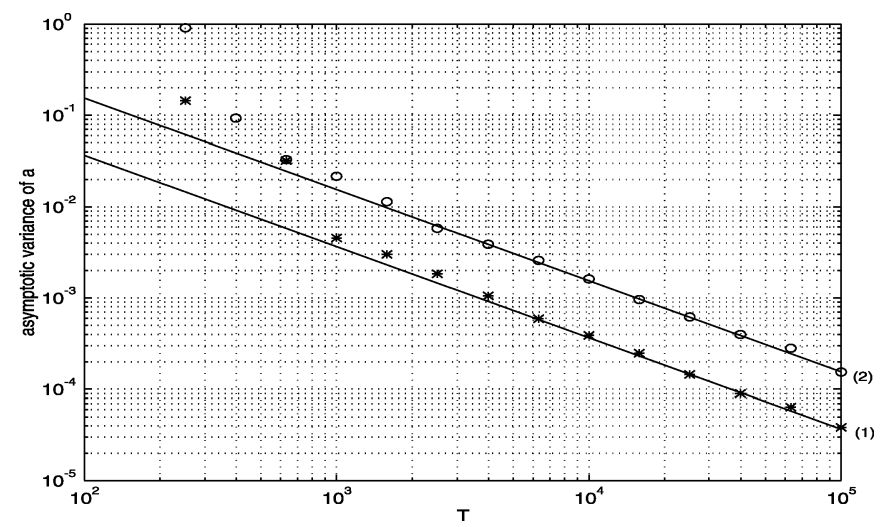

(b)

Fig. 6. (a) Third-order theoretical asymptotic lower bound and empirical (averaged on 1000 independent Monte Carlo runs) asymptotic variance given by the AMV3 (1) and $C(k, q)(2)$ algorithms as a function of $T$, for $a=0.5$, $b=0.5$, and SNR $=0 \mathrm{~dB}$. (b) SNR $=20 \mathrm{~dB}$.

In the second experiment, an $\mathrm{MA}(2)$ process is considered, where $\Theta_{1}=\left(a_{1}, a_{2}\right)^{T}$ and $\Theta_{2}=\kappa_{3_{u}}$. Fig. 7(a) [resp. (7b)] shows the normalized asymptotic lower bound and theoretical variance given by the $C(k, q)$ algorithm for the estimated parameters $a_{1}$ [resp. $a_{2}$ ] as a function of the parameters $a_{2}$ [resp. $\left.a_{1}\right]$ of the MA(2) process $x_{t}$. As for the MA(1) case, we see that the performance is very sensitive to the value of the MA parameters. As for MA(1), we see that the performance is very sensitive to the spectrum of $x_{t}$. We note that contrary to the MA(1) case, no algorithm is adequate to estimate a parameter $a_{2}$ close to zero. Furthermore, the performance of the $C(k, q)$ algorithm is practically uniformly optimal among the class of third-order-based algorithms.

In the third experiment, the processes are complex valued, the input $u_{t}$ is an 8-PSK modulation with power $\sigma_{u}^{2}$ and $\kappa_{4_{u}}=$ $-\sigma_{u}^{4}, \kappa_{6_{u}}=4 \sigma_{u}^{6}, \kappa_{8_{u}}=-33 \sigma_{u}^{810}$ and $\epsilon_{t}$ is either complex circular Gaussian i.i.d. or $\operatorname{AR}(1)\left(\epsilon_{t}=\epsilon_{t-1}+b e_{t}\right.$, where $e_{t}$ complex circular Gaussian i.i.d.).

Figs. 8 and 9 show the normalized lower bound for the asymptotic variance $\left(\operatorname{Tr}\left(\mathbf{C}_{\Theta_{1}}\right)\right)$ of estimates of $a(a=$ $\left.0.5 e^{i(\pi / 4)}\right)$ based on the sample fourth-order cumulants $\mathbf{c}^{y}(T) \stackrel{\text { def }}{=}\left\{c_{0,0,0}^{y}(T), c_{0,0,1}^{y}(T), c_{0,1,0}^{y}(T), c_{0,1,1}^{y}(T)\right.$,

${ }^{10}$ Because for up to fourth-order circular processes $\kappa_{6 u}=\mathrm{E}\left|u_{t}^{6}\right|-$ $9 \mathrm{E}\left|u_{t}^{4}\right| \mathrm{E}\left|u_{t}^{2}\right|+2 \times 6\left(\mathrm{E}\left|u_{t}^{2}\right|\right)^{3}$ and $\kappa_{8 u}=\mathrm{E}\left|u_{t}^{8}\right|-16 \mathrm{E}\left|u_{t}^{6}\right| \mathrm{E}\left|u_{t}^{2}\right|-$ $18 \mathrm{E}\left|u_{t}^{4}\right| \mathrm{E}\left|u_{t}^{4}\right|+2 \times 72 \mathrm{E}\left|u_{t}^{4}\right|\left(\mathrm{E}\left|u_{t}^{2}\right|\right)^{2}-6 \times 24\left(\mathrm{E}\left|u_{t}^{2}\right|\right)^{4}$.

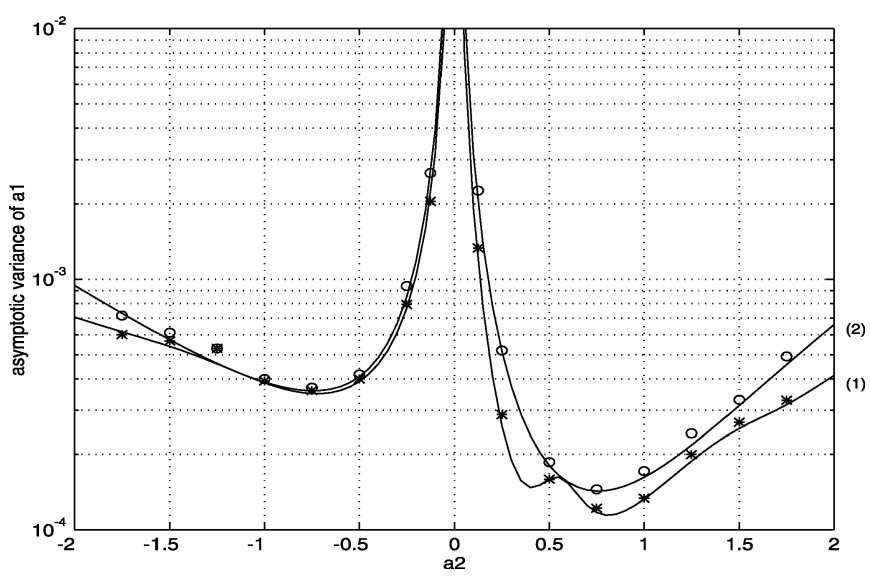

(a)

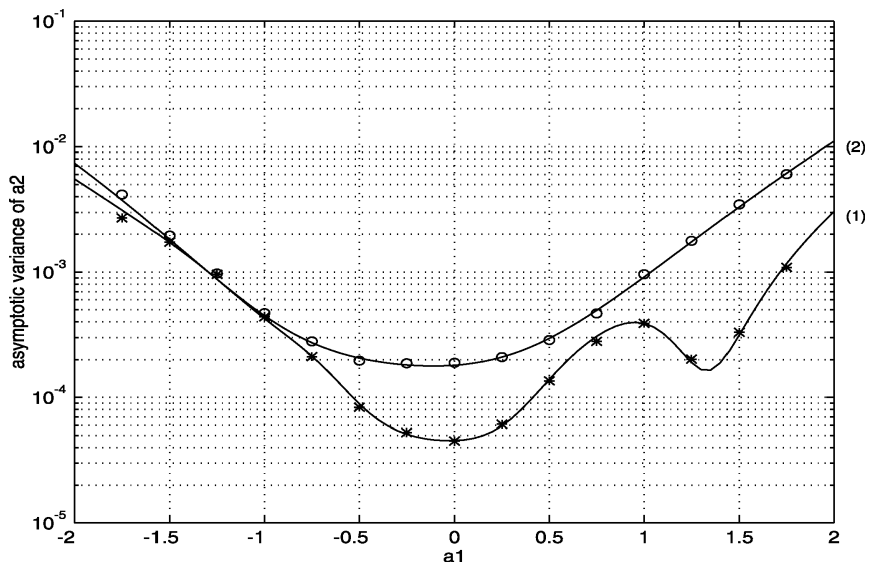

(b)

Fig. 7. Third-order theoretical asymptotic lower bound and empirical (averaged on 1000 independent Monte Carlo runs) asymptotic variance given by the AMV3 and $C(k, q)$ algorithms for the estimated parameter (a) $a_{1}$ as a function of $a_{2}$ for $a_{1}=0.8, b=0.5, \mathrm{SNR}=10 \mathrm{~dB}$, and $T=10^{5}$. (b) $a_{2}$ as a function of $a_{1}$ for $a_{2}=0.5, b=0.5, \mathrm{SNR}=10 \mathrm{~dB}$, and $T=10^{5}$.

$\left.c_{1,0,1}^{y}(T), c_{1,1,1}^{y}(T)\right\}^{11}$ and the normalized asymptotic variances given by the so-called $C(k, q)$ formula (1) extended to the complex case and by the following modified $C(k, q)$ formulae (2)(3):

- $\left.a(T)=c_{0,1,1}^{y}(T) / c_{0,0,1}^{y}(T)\right)(1)$;

- $\quad a(T)=c_{1,0,1}^{y}(T)^{*} / c_{0,0,1}^{y}(T)^{*}$ because $c_{1,0,1}=\kappa_{4_{u}} a^{* 2}$ and $c_{0,0,1}=\kappa_{4_{u}} a^{*}(2)$;

- $\quad a(T)=c_{1,1,1}^{y}(T) / c_{1,0,1}^{y}(T)$ because $c_{1,1,1}=\kappa_{4_{u}} a^{*}|a|^{2}$ and $c_{1,0,1}=\kappa_{4_{u}} a^{* 2}(3)$

for white and $\mathrm{AR}(1)$ noise $(b=0.999)$ as a function of the SNR. We note that here, $\Theta_{1}=(\Re(a), \Im(a))^{T}$ and $\Theta_{2}=\kappa_{4_{u}}$. Similar to the third-order case, we see from these two figures that beyond a certain threshold, the asymptotic lower bound and the asymptotic variance of the estimate of $a$ grows rapidly with the noise level. Beyond this threshold (for about SNR $>15 \mathrm{~dB}$ ), the asymptotic variance is approximately constant. Furthermore, we see that below this threshold, the asymptotic variances given by

\footnotetext{
${ }^{11}$ Because $c_{k_{1}, k_{2}, k_{3}}^{y} \stackrel{\text { def }}{=} \operatorname{Cum}\left(y_{0}, y_{k_{1}}^{*}, y_{k_{2}}, y_{k_{3}}^{*}\right)$, we note that $c_{0,0,0}^{y}(T)$ , $c_{0,1,1}^{y}(T) \stackrel{{ }^{2}}{=} c_{1,1,0}^{y}(T)$ are real valued, and $c_{0,0,1}^{y}(T)^{=}=$ $c_{1,0,0}^{y}(T)=c_{0,1,0}^{y}(T)$ are complex valued. Consequently, the statistic $c^{y}(T)$ used for the AMV4 estimator is composed of $\left\{c_{0,0,0}^{y}(T), c_{0,1,1}^{y}(T), c_{0,0,1}^{y}(T), c_{1,0,1}^{y}(T), c_{1,1,1}^{y}(T), c_{0,0,1}^{y} *(T), c_{1,0,1}^{y}{ }^{*}\right.$ $\left.(T), c_{1,1,1}^{y} *(T)\right\}^{1}$
} 


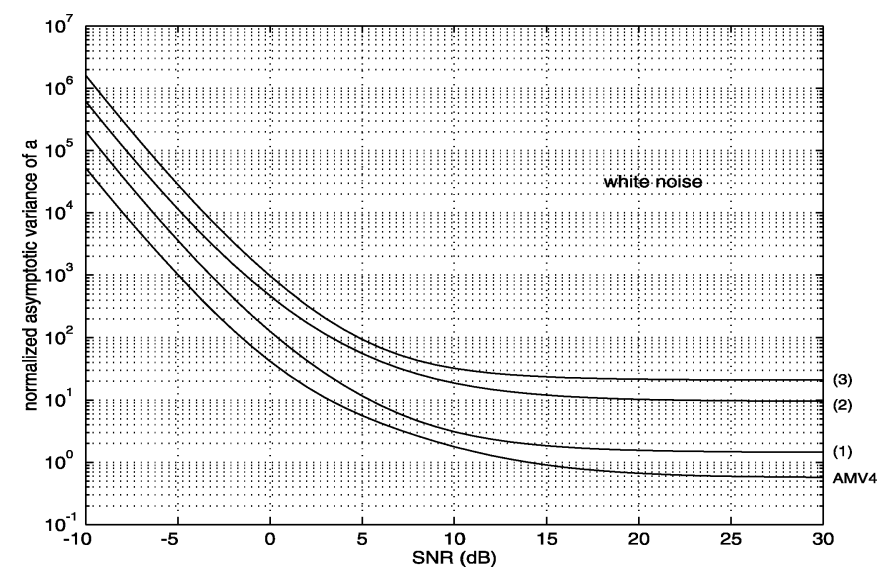

Fig. 8. Fourth-order normalized asymptotic lower bound (AMV4) and variances given by the $C(k, q)$ formulae (1), (2), and (3) as a function of the SNR.

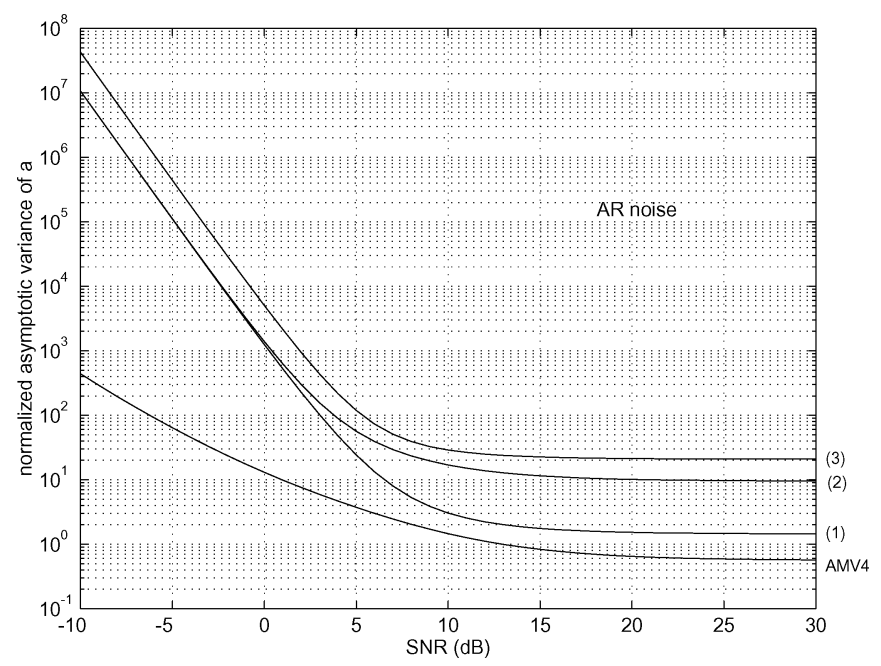

Fig. 9. Fourth-order normalized asymptotic lower bound (AMV4) and variances given by the $C(k, q)$ formulae (1), (2), and (3) as a function of the SNR.

all the $C(k, q)$ algorithms grow more rapidly than in the third order with the noise level as anticipated from the relation (6.3) extended to the fourth-order case

$$
\begin{aligned}
\lim _{T \rightarrow \infty} T \operatorname{Var}(a(T))=v_{0}+\left[\beta_{1}\left(\frac{\sigma_{\epsilon}^{2}}{\sigma_{x}^{2}}\right)\right. & +\beta_{2}\left(\frac{\sigma_{\epsilon}^{4}}{\sigma_{x}^{4}}\right) \\
& \left.+\beta_{3}\left(\frac{\sigma_{\epsilon}^{6}}{\sigma_{x}^{6}}\right)+\beta_{4}\left(\frac{\sigma_{\epsilon}^{8}}{\sigma_{x}^{8}}\right)\right] .
\end{aligned}
$$

We see that the $C(k, q)$ formula outperforms the modified $C(k, q)$ formulae, except for small SNR where their performance is similar. Furthermore, we note that contrary to all the $C(k, q)$ formulae whose performance degrades for $\mathrm{AR}(1)$ noise compared to white noise of the same power, the AMV estimate improves for small SNR. Consequently, there must be fourth-order algorithms that are much more efficient than the $C(k, q)$ formulae for small SNR.

Fig. 10 shows the normalized asymptotic lower bound based on $\mathbf{c}^{y}(T)$ (AMV4), the normalized asymptotic lower bound based on the statistics used in the $C(k, q)$ formula (1), and the modified $C(k, q)$ formula (2), i.e., based on $\mathbf{c}^{y^{\prime}}(T) \stackrel{\text { def }}{=}\left\{c_{0,1,1}^{y}\right.$

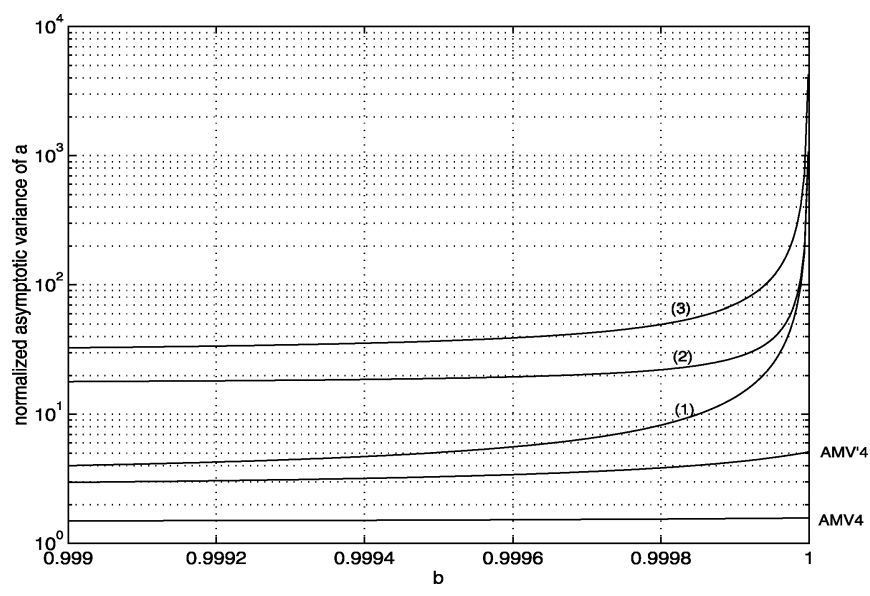

Fig. 10. Fourth-order normalized asymptotic lower bounds (AMV4) (AMV4') and normalized asymptotic variance given by the $C(k, q)$ formulae $(1),(2)$, and (3) as a function of the noise parameter $b$ for $a=0.5 e^{i(\pi / 4)}$ and SNR $=10 \mathrm{~dB}$.

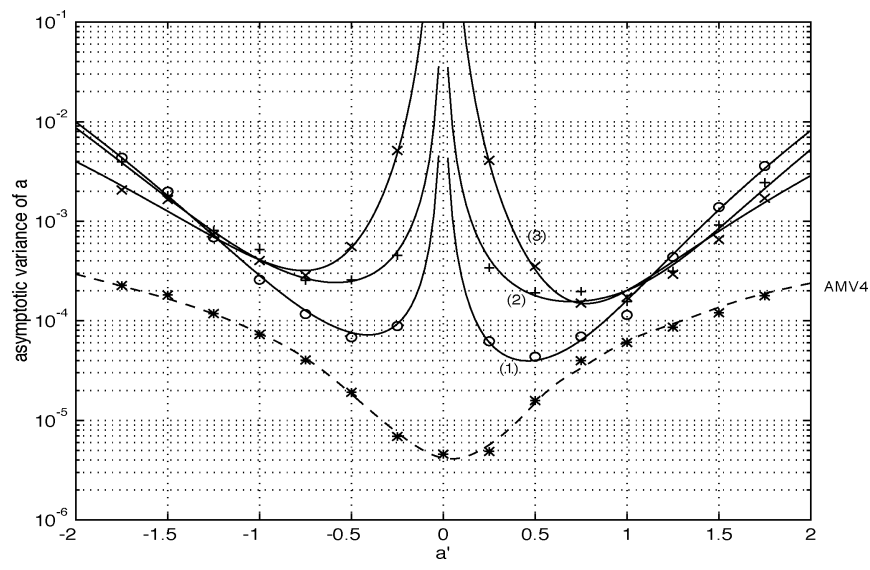

Fig. 11. Fourth-order asymptotic lower bound (AMV4) and asymptotic theoretical and empirical (averaged on 100 independent Monte Carlo runs) variance given by the $C(k, q)$ formulae (1), (2), and (3) as a function of the MA parameter $a^{\prime}$ for $b=0.999, \mathrm{SNR}=10 \mathrm{~dB}$, and $T=10^{5}$.

$\left.(T), c_{0,0,1}^{y}(T), c_{1,0,1}^{y}(T), c_{0,0,1}^{y}{ }^{*}(T), c_{1,0,1}^{y}{ }^{*}(T)\right\}$ (AMV4') and the normalized asymptotic variance given by the $C(k, q)$ formula (1) and by the modified $C(k, q)$ formulae (2) and (3) as a function of the parameter $b$ of the AR(1) noise $\epsilon_{t}$. Because these variances are relatively constant and symmetric w.r.t. zero, we focus on the [0.999 1] domain of $b$, where only the performance of the $C(k, q)$ formulae degrades when $b$ approaches \pm 1 . The AMV4 and the AMV4' are relatively insensitive to the spectrum of the additive noise $\epsilon_{t}$, including in the immediate vicinity of \pm 1 .

Fig. 11 shows the asymptotic lower bound based on $c^{y}(T)$ (AMV4) and the asymptotic theoretical and empirical variance given by the $C(k, q)$ formula (1), by the modified $C(k, q)$ formulae (2) and (3) as a function of the parameter $a^{\prime}$ $\left[a=a^{\prime} e^{i(\pi / 4)}\right.$ with $\left.a^{\prime} \in(-2,+2)\right]$ of the MA(1) process $x_{t}$. We see that the performance is very sensitive to the spectrum of the MA(1) process $x_{t}$. Furthermore, we note that there is no uniformly minimum variance estimator among the three $C(k, q)$ formulae that are inadequate to estimate a parameter $a$ close to zero.

Finally, Fig. 12 shows the normalized asymptotic lower bound for the asymptotic variance of estimates of $a$ 


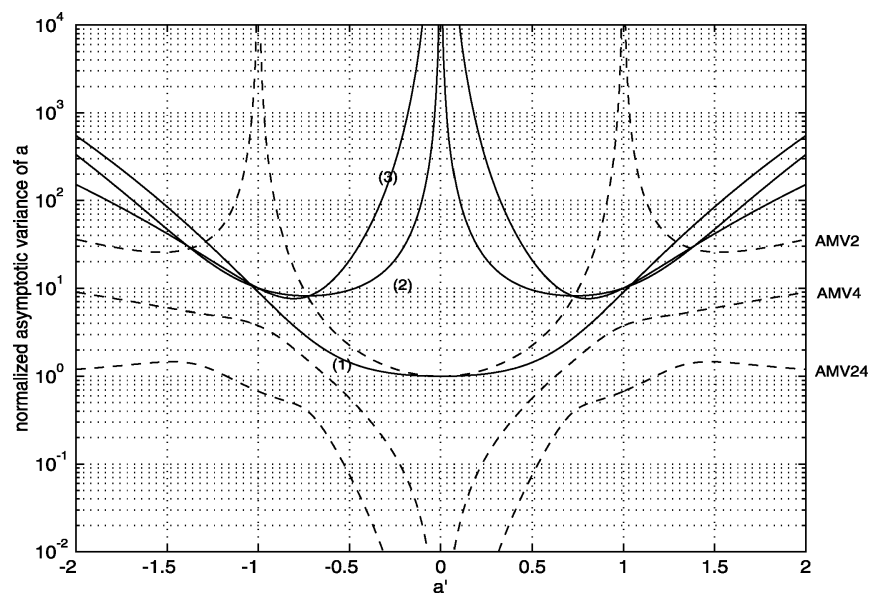

Fig. 12. Fourth-order normalized asymptotic lower bound based on sample covariances only (AMV2), on sample fourth-order cumulants only (AMV4), and on sample covariances and fourth-order cumulants (AMV24) and normalized asymptotic variance given by the $C(k, q)$ formulae (1), (2), and (3) as a function of the MA parameter $a^{\prime}$.

computed for three different cases: when the statistic $\mathbf{c}^{y}(T)$ consists of the sample covariances only ${ }^{12}$ $\left\{c_{0}^{y}(T), c_{1}^{y}(T), c_{1}^{y}(T)^{*}\right\} \quad$ (AMV2), when the statistic $\mathbf{c}^{y}(T)$ consists of the sample fourth-order cumulants only $\left\{c_{0,0,0}^{y}(T), c_{0,1,1}^{y}(T), c_{0,0,1}^{y}(T), c_{1,0,1}^{y}(T), c_{1,1,1}^{y}(T), c_{0,0,1}^{y}{ }^{*}(T)\right.$, $\left.c_{1,0,1}^{y}(T), c_{1,1,1}^{y}(T)\right\}$ (AMV4) and when the statistic $\mathbf{c}^{y}(T)$ consists of the preceding sample covariances and sample fourth-order cumulants (AMV24). This figure also exhibits the normalized asymptotic variance given by the $C(k, q)$ formulae (1)(2)(3). In order for the parameter $a$ to be identifiable from the sample covariance only, this figure is drawn in the noise-free case. As we see from this figure, there is a considerable amount of information in the fourth-order sample cumulants compared to the information in the sample covariances. Furthermore, we note that contrary to the noisy case, the AMV vanishes for a parameter $a$ close to zero.

Naturally, these examples are totally inadequate for predicting the asymptotic performance of a specific third- or fourth-order algorithm in the presence of additive colored noise. They simply show the potentially large sensitivity of the asymptotic performance of an arbitrary algorithm to the spectra of the linear process and of the colored noise.

\section{CONCLUSION}

This paper has extended Bartlett's formula to the third and fourth order and to noisy linear processes, thanks to a polyspectral approach and a symbolic calculus akin to a high-level language. As an application of these closed-form expressions, the sensitivity of the asymptotic performance of the estimated ARMA parameters by an arbitrary third- or fourth-order-based algorithm to the SNR, the spectra of the linear process, and the colored additive noise is addressed. Such sensitivity analysis has been possible, thanks to the numerical expressions derived

\footnotetext{
${ }^{12}$ As estimation methods based on the sample covariances only cannot distinguish between nonminimum phase and minimum phase processes having the same spectrum, the bound for this case applies only to estimators that are based on prior knowledge of the zero locations within a sufficiently small error.
}

from our theoretical expressions, whereas Monte Carlo simulations have accommodated only particular scenarios until now. As an example, the asymptotic lower bound for the variances of third- or fourth-order algorithms are compared to the asymptotic variances given by the so-called $C(k, q)$ algorithms for non-Gaussian first- or second-order MA processes corrupted by a Gaussian first-order AR process with respect to the SNR and to the MA and AR parameters. In particular, we have shown that the performance presents a threshold effect with respect to the SNR and is very sensitive to the spectrum of the MA process but relatively insensitive to the AR spectrum, except for sharp resonances.

\section{APPENDIX A}

\section{PROOF OF EQUATION (4.5)}

Because $\int_{\triangle} e^{i 2 \pi f \tau} d f=1$ if $\tau=0$ and $\int_{\triangle} e^{i 2 \pi f \tau} d f=0$ if $\tau \neq 0$

$$
\begin{aligned}
\sum_{t} & \operatorname{Cum}\left(x_{0}, x_{k}, x_{t}, x_{t+l}, x_{t+m}\right) \\
= & \int_{\triangle^{3}} \sum_{t_{1}} \sum_{t} \sum_{t_{2}} \sum_{t_{3}} c_{t_{1}, t, t_{2}, t_{3}}^{x} \\
& \times e^{-i 2 \pi\left[f_{1}\left(t_{1}-k\right)+f_{2}\left(t_{2}-t-l\right)+f_{3}\left(t_{3}-t-m\right)\right]} d f_{1} d f_{2} d f_{3} \\
= & \int_{\triangle^{3}} S_{x}\left(f_{1},-f_{2}-f_{3}, f_{2}, f_{3}\right) e^{i 2 \pi\left[f_{1} k+f_{2} l+f_{3} m\right]} d f_{1} d f_{2} d f_{3}
\end{aligned}
$$

and $S_{x}\left(f_{1},-f_{2}-f_{3}, f_{2}, f_{3}\right)=S_{x}\left(f_{1},-f_{1}, f_{2}, f_{3}\right)$ from [4].

Then, looking at each term of $\sum_{t} \operatorname{Cum}\left(x_{0}, x_{k}, x_{t}\right)$ $\operatorname{Cum}\left(x_{t+l}, x_{t+m}\right)$ [10], there appears a term $c_{k}^{x} c_{l, m}^{x}$ that vanishes in (4.1), three terms of the form $\sum_{t} c_{\alpha}^{x} c_{\beta, t+\gamma}^{x}$ and six terms of the form $\sum_{t} c_{t+\alpha}^{x} c_{-t+\beta, \gamma}^{x}$, which become, respectively,

$$
\begin{aligned}
& \sum_{t} c_{\alpha}^{x} c_{\beta, t+\gamma}^{x} \\
& =c_{\alpha}^{x} \int_{\triangle} \sum_{t_{1}} \sum_{t} c_{t_{1}, t}^{x} e^{-i 2 \pi\left[f_{1}\left(t_{1}-\beta\right)+0 t\right]} d f_{1} \\
& =c_{\alpha}^{x} \int_{\triangle} S_{x}\left(f_{1}, 0\right) e^{i 2 \pi f_{1} \beta} d f_{1} \\
& =\int_{\triangle^{2}} S_{x}\left(f_{1}, 0\right) S_{x}\left(f_{2}\right) e^{i 2 \pi\left[f_{1} \beta+f_{2} \alpha\right]} d f_{1} d f_{2}, \\
& \sum_{t} c_{t+\alpha}^{x} c_{-t+\beta, \gamma}^{x} \\
& =\int_{\triangle}\left[S_{x}(f) \int_{\triangle} S_{x}\left(f, f_{1}\right) e^{i 2 \pi\left[f \beta+f_{1} \gamma\right]} d f_{1}\right] e^{i 2 \pi f \alpha} d f \\
& =\int_{\triangle^{2}} S_{x}\left(f_{1}\right) S_{x}\left(f_{1}, f_{2}\right) e^{i 2 \pi\left[f_{1}(\alpha+\beta)+f_{2} \gamma\right]} d f_{1} d f_{2}
\end{aligned}
$$

because the second term is a convolution product of $c_{\tau}^{x}$ and $c_{\tau+\beta, \gamma}^{x}$ at the point $\tau=\alpha$, since $\int_{\triangle} S_{x}\left(f, f_{1}\right) e^{i 2 \pi\left[f \beta+f_{1} \gamma\right]} d f_{1}$ is the Fourier transform of the sequence $\left(c_{\tau+\beta, \gamma}^{x}\right)_{\tau \in Z}$. Combining these groups of terms, the matrix expression (4.5) is obtained. 


\section{APPENDIX B \\ EXPRESSIONS OF THE DIFFERENT MATRICES $\mathbf{E}_{j}^{(i)}$ OF SECTION IV}

Our symbolic algorithm gives rels. (4.5) and (4.6), where matrices $\mathbf{E}_{j}^{(i)}$ are composed of finite sums of $e^{i 2 \pi\left(f_{1} a_{1}+f_{2} a_{2}+f_{3} a_{3}\right)}$ defined by the following:

$$
\begin{aligned}
& \mathbf{E}_{1,2,3} \stackrel{\text { def }}{=} \mathbf{e}_{1}\left(\mathbf{e}_{2}^{T} \otimes \mathbf{e}_{3}^{T}\right) \\
& \mathbf{E}_{1,2}^{(1)} \stackrel{\text { def }}{=} \mathbf{e}_{1}^{*}\left(\mathbf{e}_{2}^{T} \otimes \mathbf{e}_{1}^{T}+\mathbf{e}_{1}^{T} \otimes \mathbf{e}_{2}^{T}+\mathbf{e}_{1,2}^{H} \otimes \mathbf{e}_{2}^{T}\right) \\
& +\mathbf{e}_{1}\left(\mathbf{e}_{2}^{T} \otimes \mathbf{e}_{1}^{T}+\mathbf{e}_{1}^{T} \otimes \mathbf{e}_{2}^{T}+\mathbf{e}_{1,2}^{H} \otimes \mathbf{e}_{2}^{T}\right) \\
& \mathbf{E}_{1,2}^{(2)} \stackrel{\text { def }}{=} \mathbf{e}_{1}\left(\mathbf{e}_{2}^{T} \otimes \mathbf{e}_{2}^{H}+\mathbf{e}_{0}^{T} \otimes \mathbf{e}_{2}^{T}+\mathbf{e}_{2}^{T} \otimes \mathbf{e}_{0}^{T}\right) \\
& \mathbf{E}_{1,2,3,4} \stackrel{\text { def }}{=} \mathbf{e}_{1} \mathbf{e}_{3}^{T} \otimes \mathbf{e}_{2} \mathbf{e}_{4}^{T} \\
& \mathbf{E}_{1,2,3}^{(1)} \stackrel{\text { def }}{=} \mathbf{e}_{2} \mathbf{e}_{1}^{T} \otimes \mathbf{e}_{2,3}^{*} \mathbf{e}_{3}^{T}+\mathbf{e}_{2} \mathbf{e}_{3}^{T} \otimes \mathbf{e}_{2,3}^{*} \mathbf{e}_{1}^{T} \\
& +\mathbf{e}_{1} \mathbf{e}_{2}^{T} \otimes \mathbf{e}_{3} \mathbf{e}_{2,3}^{H}+\mathbf{e}_{3} \mathbf{e}_{2}^{T} \otimes \mathbf{e}_{1} \mathbf{e}_{2,3}^{H} \\
& +\mathbf{e}_{1} \mathbf{e}_{3}^{T} \otimes \mathbf{e}_{3} \mathbf{e}_{2}^{T}+\mathbf{e}_{1} \mathbf{e}_{2}^{T} \otimes \mathbf{e}_{3} \mathbf{e}_{3}^{T} \\
& +\mathbf{e}_{3} \mathbf{e}_{3}^{T} \otimes \mathbf{e}_{1} \mathbf{e}_{2}^{T}+\mathbf{e}_{3} \mathbf{e}_{2}^{T} \otimes \mathbf{e}_{1} \mathbf{e}_{3}^{T} \\
& +\mathbf{e}_{2,3}^{*} \mathbf{e}_{1,3}^{H} \otimes \mathbf{e}_{2} \mathbf{e}_{1}^{T} \\
& \mathbf{E}_{1,2,3}^{(2)} \stackrel{\text { def }}{=} \mathbf{e}_{3} \mathbf{e}_{1}^{T} \otimes \mathbf{e}_{3}^{*} \mathbf{e}_{2}^{T}+\mathbf{e}_{0} \mathbf{e}_{1}^{T} \otimes \mathbf{e}_{3} \mathbf{e}_{2}^{T}+\mathbf{e}_{3} \mathbf{e}_{1}^{T} \otimes \mathbf{e}_{0} \mathbf{e}_{2}^{T} \\
& +\mathbf{e}_{1} \mathbf{e}_{3}^{T} \otimes \mathbf{e}_{2} \mathbf{e}_{3}^{H}+\mathbf{e}_{1} \mathbf{e}_{3}^{T} \otimes \mathbf{e}_{2} \mathbf{e}_{0}^{T}+\mathbf{e}_{1} \mathbf{e}_{0}^{T} \otimes \mathbf{e}_{2} \mathbf{e}_{3}^{T} \\
& \mathbf{E}_{1,2,3}^{(3)} \stackrel{\text { def }}{=} \mathbf{e}_{1} \mathbf{e}_{2,3}^{H} \otimes \mathbf{e}_{2,3} \mathbf{e}_{3}^{T}+\mathbf{e}_{1} \mathbf{e}_{3}^{T} \otimes \mathbf{e}_{2,3} \mathbf{e}_{2,3}^{H} \\
& +\mathbf{e}_{1} \mathbf{e}_{2}^{T} \otimes \mathbf{e}_{2,3} \mathbf{e}_{3}^{T}+\mathbf{e}_{2,3} \mathbf{e}_{2,3}^{H} \otimes \mathbf{e}_{1} \mathbf{e}_{3}^{T} \\
& +\mathbf{e}_{2,3} \mathbf{e}_{3}^{T} \otimes \mathbf{e}_{1} \mathbf{e}_{2,3}^{H}+\mathbf{e}_{2,3} \mathbf{e}_{2}^{T} \otimes \mathbf{e}_{1} \mathbf{e}_{3}^{T} \\
& +\mathbf{e}_{1} \mathbf{e}_{2}^{T} \otimes \mathbf{e}_{1,2,3}^{*} \mathbf{e}_{3}^{T}+\mathbf{e}_{1} \mathbf{e}_{2,3}^{H} \otimes \mathbf{e}_{1,2,3}^{*} \mathbf{e}_{3}^{T} \\
& +\mathbf{e}_{1} \mathbf{e}_{3}^{T} \otimes \mathbf{e}_{1,2,3}^{*} \mathbf{e}_{2,3}^{H} \\
& \mathbf{E}_{1,2}^{(3)} \stackrel{\text { def }}{=} \mathbf{e}_{1} \mathbf{e}_{2}^{T} \otimes \mathbf{e}_{0} \mathbf{e}_{2}^{H}+\mathbf{e}_{1} \mathbf{e}_{0}^{T} \otimes \mathbf{e}_{0} \mathbf{e}_{2}^{T}+\mathbf{e}_{1} \mathbf{e}_{2}^{T} \otimes \mathbf{e}_{0} \mathbf{e}_{0}^{T} \\
& +\mathbf{e}_{0} \mathbf{e}_{2}^{T} \otimes \mathbf{e}_{1} \mathbf{e}_{2}^{H}+\mathbf{e}_{0} \mathbf{e}_{0}^{T} \otimes \mathbf{e}_{1} \mathbf{e}_{2}^{T}+\mathbf{e}_{0} \mathbf{e}_{2}^{T} \otimes \mathbf{e}_{1} \mathbf{e}_{0}^{T} \\
& +\mathbf{e}_{1} \mathbf{e}_{2}^{T} \otimes \mathbf{e}_{1}^{*} \mathbf{e}_{2}^{H}+\mathbf{e}_{1} \mathbf{e}_{0}^{T} \otimes \mathbf{e}_{1}^{*} \mathbf{e}_{2}^{T}+\mathbf{e}_{1} \mathbf{e}_{2}^{T} \otimes \mathbf{e}_{1}^{*} \mathbf{e}_{0}^{T} \\
& \mathbf{E}_{1,2}^{(4)} \stackrel{\text { def }}{=} \mathbf{e}_{2}^{*} \mathbf{e}_{2}^{T} \otimes \mathbf{e}_{1,2} \mathbf{e}_{1,2}^{H}+\mathbf{e}_{2}^{*} \mathbf{e}_{1,2}^{H} \otimes \mathbf{e}_{1,2} \mathbf{e}_{2}^{T} \\
& +\mathbf{e}_{2}^{*} \mathbf{e}_{1}^{T} \otimes \mathbf{e}_{1,2} \mathbf{e}_{1,2}^{H}+\mathbf{e}_{1,2} \mathbf{e}_{1}^{T} \otimes \mathbf{e}_{2}^{*} \mathbf{e}_{1,2}^{H} \\
& +\mathbf{e}_{2}^{*} \mathbf{e}_{1,2}^{H} \otimes \mathbf{e}_{1,2} \mathbf{e}_{1}^{T}+\mathbf{e}_{1,2} \mathbf{e}_{1,2}^{H} \otimes \mathbf{e}_{2}^{*} \mathbf{e}_{1}^{T}
\end{aligned}
$$

where $\mathbf{e}_{0} \stackrel{\text { def }}{=}(1, \ldots, 1)^{T}, \mathbf{e}_{k} \stackrel{\text { def }}{=}\left(1, e^{i 2 \pi f_{k}}, \ldots, e^{i 2 \pi(L-1) f_{k}}\right)^{T}$ and $\mathbf{e}_{k, l} \stackrel{\text { def }}{=}\left(1, e^{i 2 \pi\left(f_{k}+f_{l}\right)}, \ldots, e^{i 2 \pi(L-1)\left(f_{k}+f_{l}\right)}\right)^{T}, \mathbf{e}_{k, l, m} \stackrel{\text { def }}{=}$ $\left(1, e^{i 2 \pi\left(f_{k}+f_{l}+f_{m}\right)}, \ldots, e^{i 2 \pi(L-1)\left(f_{k}+f_{l}+f_{m}\right)}\right)^{T}, k, l, m=1,2$, 3, ( $L=L_{2}$ in the first three equations and $L=L_{3}$ elsewhere).

\section{APPENDIX C}

PROOF OF EQUATION (4.9)

Because $S_{\epsilon}\left(f_{1}\right)=\sum_{k} \sigma_{\epsilon}^{2} b^{|k|} e^{-i 2 \pi k f_{1}}$

$$
\begin{aligned}
& \int_{\triangle^{2}} S_{\epsilon}\left(f_{1}\right) S_{\epsilon}\left(f_{2}\right) S_{\epsilon}\left(f_{1}+f_{2}\right) d f_{1} d f_{2} \\
& =\int_{\triangle^{2}} \sum_{k} \sum_{l} \sum_{m} \sigma_{\epsilon}^{6} b^{|l|} b^{|k|} b^{|m|} e^{-i 2 \pi\left[(k+m) f_{1}+(l+m) f_{2}\right]} d f_{1} d f_{2} \\
& =\sum_{m} \sigma_{\epsilon}^{6} b^{|3 m|}=\sigma_{\epsilon}^{6}\left(1+2 \sum_{m=1}^{\infty} b^{3 k}\right)=\sigma_{\epsilon}^{6}\left(\frac{1+b^{3}}{1-b^{3}}\right) .
\end{aligned}
$$

\section{REFERENCES}

[1] D. R. Brillinger and M. Rosenblatt, "Asymptotic theory of $k$-order spectra," in Spectral Analysis of Times Series, B. Harris, Ed. New York: Wiley, 1967, pp. 153-188.

[2] D. R. Brillinger, Times Series; Data Analysis and Theory. San Francisco, CA: Holden Day, 1981.

[3] G. B. Giannakis, "Cumulants: A powerful tool in signal processing," Proc. IEEE, vol. 75, no. 9, pp. 1333-1334, Sep. 1987.

[4] J. M. Mendel, "Tutorial on higher-order statistics (spectra) in signal processing and system theory: theoretical results and some applications," Proc. IEEE, vol. 79, no. 3, pp. 278-305, Mar. 1991.

[5] B. Porat and B. Friedlander, "Performance analysis of parameter estimation algorithms based on high-order moments," Int. J. Adaptive Control, Signal Process., vol. 3, pp. 191-229, 1989.

[6] G. B. Giannakis and J. M. Mendel, "Identification of nonminimum phase system using higher order statistics," IEEE Trans. Acoust., Speech, Signal Process., vol. 37, no. 3, pp. 360-376, Mar. 1989.

[7] J. K. Tugnait, "New results on FIR system identification using higher order statistics," IEEE Trans. Signal Process., vol. 39, no. 10, pp. 2216-2221, Oct. 1991.

[8] X. D. Zhang and Y. S. Zhang, "FIR system identification using higher order statistics alone," IEEE Trans. Signal Process., vol. 42, no. 10, pp. 2854-2858, Oct. 1994.

[9] A. V. Dandawaté and G. B. Giannakis, "Asymptotic properties and covariance expressions of $k$-th-order sample moments and cumulants," in Proc. Asilomar Conf. Signals, Syst., Comput., Pacific Grove, CA, Nov. 1993, pp. 1186-1190.

[10] - "Asymptotic theory of mixed time averages and $k$-th-order cyclicmoment and cumulant statistics," IEEE Trans. Inf. Theory, vol. 41, no. 1, pp. 216-232, Jan. 1995.

[11] M. Rosenblatt, Stationary Sequences and Random Fields. Cambridge, MA: Birkhäuser, 1985.

[12] M. S. Bartlett, An Introduction of Stochastic Process, 2nd ed. Cambridge, U.K.: Cambridge Univ. Press, 1966.

[13] B. Porat, Digital Processing of Random Signals, Theory and Methods. Englewood Cliffs, NJ: Prentice-Hall, 1994.

[14] P. McCullagh, Tensor Methods in Statistics. London, U.K.: Chapman \& Hall, 1986.

[15] T. Söderström and P. Stoica, System Identification. Englewood Cliffs, NJ: Prentice-Hall, 1989.

[16] J. P. Delmas, "Asymptotically minimum variance second-order estimation for noncircular signal with application to DOA estimation," IEEE Trans. Signal Process., vol. 52, no. 5, pp. 1235-1241, May 2004.

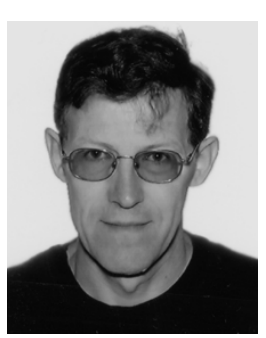

Jean Pierre Delmas (M'00) was born in France in 1950. He received the Engineering degree from Ecole Centrale de Lyon, Lyon, France, in 1973, the Certificat d'études supérieures from the Ecole Nationale Supérieure des Télécommunications, Paris, France, in 1982, and the Habilitation à diriger des recherches (HDR) degree from the University of Paris, Orsay, France, in 2001.

Since 1980, he has been with the Institut National des Télécommunications, Evry, France, where he is presently a Professor in the CITI department within UMR-CNRS 5157. His teaching and research interests are in the areas of statistical signal processing with application to communications and antenna array.

Dr. Delmas is currently an Associate Editor for the IEEE TRANSACTIONS ON SIGNAL PROCESSING.

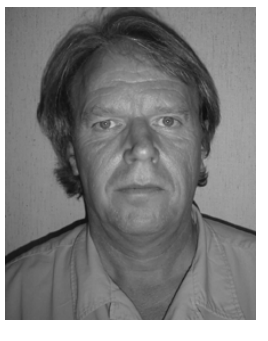

Yann Meurisse received the Engineer degree in telecommunications from the Institut National des Télécommunications (INT), Evry, France, in 1983 and the Diplôme d'Etudes Approfondies in automatic control and signal processing and the Ph.D. degree from the University of Paris Sud, Orsay, France, in 1987 and 2002, respectively.

Since 1985, he has been with INT, where he is currently an Assistant Professor in the Signal and Image Processing Department. His research interests are in antenna array processing. 